\title{
¿a $\begin{array}{ll}\text { Research Square } & \text { They should not be considered conclusive, used to inform clinical practice, } \\ \text { or referenced by the media as validated information. }\end{array}$
}

\section{Fluoride Induced Leaky Gut and Bloom of Erysipelatoclostridium Ramosum Mediate the Exacerbation of Obesity in High-Fat-Diet Fed Mice}

\section{Guijie Chen}

Nanjing Agricultural University - Weigang Campus: Nanjing Agricultural University

\section{Yujia Peng}

Nanjing Agricultural University - Weigang Campus: Nanjing Agricultural University

\section{Yujie Huang}

Nanjing Agricultural University - Weigang Campus: Nanjing Agricultural University

\section{Minhao Xie}

Nanjing University of Finance and Economics - Xianlin Campus: Nanjing University of Finance and

Economics

\section{Zhuqing Dai}

Jiangsu Academy of Agricultural Sciences

\section{Huimei Cai}

Anhui Agriculture University: Anhui Agricultural University

\section{Wei Dong}

Nanjing Agricultural University - Weigang Campus: Nanjing Agricultural University

\section{Weiqi Xu}

Nanjing Agricultural University - Weigang Campus: Nanjing Agricultural University

\section{Zhiyong Xie}

Nanjing Agricultural University - Weigang Campus: Nanjing Agricultural University

\section{Dan Chen}

Nanjing Agricultural University - Weigang Campus: Nanjing Agricultural University

\section{Xia Fan}

Nanjing Agricultural University - Weigang Campus: Nanjing Agricultural University

\section{Wangting Zhou}

Nanjing Agricultural University - Weigang Campus: Nanjing Agricultural University

\section{Xuhui Kan}

Nanjing Agricultural University - Weigang Campus: Nanjing Agricultural University

\section{Tingting Yang}

Nanjing Agricultural University - Weigang Campus: Nanjing Agricultural University

\section{Chunxu Chen}

Nanjing Agricultural University - Weigang Campus: Nanjing Agricultural University 


\section{Yi Sun}

Nanjing Agricultural University - Weigang Campus: Nanjing Agricultural University

Xiaoxiong Zeng ( $\nabla$ zengxx@njau.edu.cn )

Nanjing Agricultural University - Weigang Campus: Nanjing Agricultural University https://orcid.org/0000-0003-2954-3896

\section{Zhonghua Liu}

Hunan Agricultural University

\section{Research Article}

Keywords: Fluoride, Exacerbation of obesity, Intestinal barrier permeability, Gut microbiota, Erysipelatoclostridium ramosum

Posted Date: December 3rd, 2021

DOI: https://doi.org/10.21203/rs.3.rs-1069250/v1

License: @ (i) This work is licensed under a Creative Commons Attribution 4.0 International License. Read Full License 


\section{Abstract}

Background: Fluoride, a necessary mineral element for our health, is widely presented in drinking water and foods. The intake of excessive fluoride showed potential risk to human health. A strong relation between fluoride exposure and obesity has been reported. However, the knowledge on the potential mechanisms on fluoride-induced obesity is still limited.

Results: In this work, we showed here that fluoride alone did not induce obesity in normal diet fed mice, whereas, it could trigger exacerbation of obesity in high-fat diet (HFD) fed mice. Fluoride impaired intestinal barrier and activated Toll-like receptor 4 (TLR4) signaling to induce obesity, which was further verified in TLR4 $\%$ mice. Furthermore, fluoride could deteriorate the gut microbiota in HFD mice. The fecal microbiota transplantation from fluoride-induced mice was sufficient to induce obesity, while the exacerbation of obesity by fluoride was blocked upon gut microbiota depletion. The fluoride-induced bloom of Erysipelatoclostridium ramosum belonged to Erysipelotrichaceae was responsible for exacerbation of obesity. In addition, a potential strategy for prevention of fluoride-induced obesity was proposed by intervention with polysaccharides from Fuzhuan brick tea.

Conclusions: Overall, these results provide the first evidence of a comprehensive cross-talk mechanism between fluoride and obesity in HFD fed mice, which is mediated by gut microbiota and intestinal barrier. E. ramosum was identified as a crucial mediator of fluoride induced obesity, which could be explored as potential target for prevention and treatment of obesity with exciting translational value.

\section{Background}

Obesity, a complex disease characterized by a high body mass index (BMI) and excess fat accumulation in adipose etc., has dramatically spread throughout the developed and developing countries in the past few decades due to the obesogenic shifts in nutritional composition, excessive intake of calories, and lack of exercise [1, 2]. It has become a leading public health problem worldwide, which may increase the risks of numerous comorbidities such as type 2 diabetes (T2DM), nonalcoholic fatty liver disease (NAFLD), and cardiovascular disease [3, 4]. In China, the latest national prevalence estimates for 20152019 showed $16.4 \%$ for obesity and $34.3 \%$ for overweight in adults ( $\geq 18$ years), respectively [5]. Although the potential mechanisms for obesity and associated metabolic diseases are still not fully understood, increasing evidence supports that the dysbiosis of gut microbiota plays a central role in the development of various obesity-induced diseases [6, 7]. The prevention and treatment of obesity were mainly focused on end organs, such as pancreas, adipose tissue, muscle and brain, due to the involvement in glucose regulation and energy balance in the past decades, whereas, the improvement of intestinal health is considered as one of the most effective therapy for obesity-induced diseases in recent years [8]. Indeed, animal and human studies have shown that the modulation of gut microbiota by antiobesogenic candidates was contributed to the improvement of the obesity and associated metabolic diseases [8-10]. 
Obesity is caused by numerous factors, including host genetics, physiological characteristics, living environment, lifestyle and dietary habits. Thereinto, the change of dietary habit, especially excessive consumption of a high-fat diet (HFD), is one of major causes of obesity and associated metabolic diseases $[11,12]$. The taxonomical and functional changes of gut microbiota were observed after intervention of HFD, which contributed to the obesity and associated metabolic diseases $[13,14]$. Furthermore, HFD could impair intestinal integrity and induce leaky gut, then, some of gut microbiota, along with toxins such as lipopolysaccharide (LPS), could enter into bloodstream, thereby induce inflammation and metabolic diseases by activating Toll-like receptor 4 (TLR4) mechanism $[15,16]$. Besides HFD, some food ingredients or food additives could damage the intestinal health, by inducing the dysbiosis of gut microbiota, impairing intestinal integrity, thereby leading to the obesity and associated metabolic diseases. For example, artificial sweeteners, food colorants and dietary emulsifiers could induce inflammation and metabolic diseases by altering the gut microbiota [17-19]. Thus, the potential risk of food-derived ingredients or additives should be caused more attentions.

Fluoride, a necessary mineral element for our health, could prevent dental cavities and be beneficial for the development of bone in appropriate dosage. In the United States, the mean decayed-missing-filled tooth (DMFT) among adolescents aged 12 years decreased from $4.0 \%$ in $1966-1970$ to $1.3 \%$ in 1988 1994 due to water fluoridation [20]. However, the excessive consumption of fluoride could lead to the detrimental effect on human body, such as endemic dental, skeletal fluorosis, oxidative stress and neurotoxicity $[21,22]$. The drinking water is one of the main resources for intake of fluoride. The high level of fluoride in drinking water has been gradually recognized as a potential risk factor to human health, thus, the World Health Organization (WHO) has stipulated that the limit of fluoride in drinking water should be no more than $1.5 \mathrm{mg} / \mathrm{L}$ [23]. Besides the drinking water, the fluoride in some foods showed potential risk to human health [24]. For example, the tea plant, that can accumulate fluoride from the air and soil, contains high content of fluoride especially in mature leaves, and it has been reported that content of fluoride in tea could be more than $2000 \mathrm{mg} / \mathrm{kg}$, which could lead to the risk of brick-tea type fluorosis [25]. Thus, the detrimental effect of fluoride on health has been widely investigated [21, 26]. Despite the fluoride induced obesity has been confirmed by animal experiment and epidemiological study $[27,28]$, the potential mechanisms of fluoride-induced obesity are still unknown. Furthermore, the role of gut homeostasis in the exacerbation of the obesity induced by fluoride is ignored. Here, the effects and potential mechanisms of fluoride on the obesity fed with normal diet (ND) or HFD were investigated in detail using mice model. The results showed that the driving intestinal barrier permeability and disturbing gut microbiota induced by fluoride were involved in the exacerbation of the obesity in HFD mice. Furthermore, the Erysipelotrichaceae was identified as the potential key bacteria contributing to detrimental effect of fluoride on obesity, and the important role of Erysipelotrichaceae in obesity was confirmed by data from a cohort American Gut Project (AGP).

\section{Methods}

\section{Mice experiments}


All animal experiment protocols were approved by the institutional Animal Ethics Committee of Experimental Animal Center of Nanjing Agricultural University (Approval number: PZ2019043, PZ2020024, PZ2020091, and NJAU.No20210425054). The animal experiments were carried out in Animal Center of Nanjing Agricultural University (SYXK<Jiangsu>2011-0037) in accordance with the Guidelines of the Ethical Committee of Experimental Animal Center of Nanjing Agricultural University and the National Guidelines for Experimental Animal Welfare. Mice were housed under specific pathogen free (SPF) conditions with free access to sterile food (irradiated) and sterile water in $12 \mathrm{~h}$ light/dark cycle. Five or six-week-old C57BL/6J male mice were purchased from Shanghai SLAC Laboratory Animal Co., Ltd. (Shanghai, China). The TIr4 ${ }^{-/}$and WT C57BL/10J male mice with five weeks old were purchased from GemPharmatech Co., Ltd. (Nanjing, China). The ND D12450J (10\% calories from fat) and HFD D12492 ( $60 \%$ calories from fat) were obtained from Research Diets, Inc. (New Brunswick, NJ, USA), whose compositions are shown in Supplementary Table 1. Before experiment, all animal underwent a 1-week acclimatization period fed with ND. The fluoride was administrated to mice by addition to drinking water, and the drinking water was refreshed twice one week. The food intake and body weight were recorded once a week during the animal experiments.

Animal protocol 1. The purpose of this first mice experiment was to evaluate the effect of fluoride on the obesity in HFD mice. 24 male C57BL/6J mice with 6 weeks of age were randomly divided into three groups including the ND, HFD, and HFD-F groups (HFD plus $50 \mathrm{mg} / \mathrm{L}$ of fluoride in drinking water) $(\mathrm{n}=8$ per group). The intervention continued for 10 weeks, and the mice were euthanized after fasting overnight at the end of the experiment.

Animal protocol 2. The purpose of this mice experiment was to confirm the effect of fluoride on the obesity in HFD mice in a separate replicate experiment. Furthermore, the potential mechanisms were investigated based on the first mice experiment, which focused on the gut microbiota and intestinal barrier permeability. 24 male C57BL/6J mice with 5 weeks of age were randomly divided into three groups including ND, HFD, and HFD-F groups ( $n=8$ per group). The intervention continued for 10 weeks. The fecal samples were collected for 16S rRNA sequencing at the last week, and the mice were euthanized after fasting overnight at the end of the experiment.

Animal protocol 3. The purpose of this mice experiment was to analyze the effect of fluoride on intestinal barrier permeability in vivo according to the previous work [67]. 16 male C57BL/6J mice with 5 weeks of age were randomly divided into two groups including HFD and HFD-F groups ( $n=8$ per group). The intervention continued for 10 weeks. At the end of the experiment, FITC-dextran solution (4 kDa (SigmaAldrich, St. Louis, MO) with dosage of $0.6 \mathrm{mg} / \mathrm{g}$ body weight) was administrated to mice by intragastric gavage after fasting for $4 \mathrm{~h}$. Then, the mice were euthanized after $3 \mathrm{~h}$ to afford plasma for measurement of level of FITC-dextran by Fluorescence Spectrophotometer at an excitation wavelength of $485 \mathrm{~nm}$ and emission wavelength of $535 \mathrm{~nm}$.

Animal protocol 4. The purpose of this mice experiment was to investigate whether the knockout of T/r4 could block the exacerbation of obesity by fluoride in HFD mice. Sixteen WT and sixteen TIr4 ${ }^{-/-}$mice 
(C57BL/10J, 5 weeks old) were randomly divided into two groups, respectively, including HFD and HFD-F (HFD plus $50 \mathrm{mg} / \mathrm{L}$ of fluoride in drinking water) groups ( $\mathrm{n}=8$ per group). The intervention continued for 8 weeks, and the mice were euthanized after fasting overnight at the end of the experiment.

Animal protocol 5. The purpose of this mice experiment was to investigate whether fluoride-induced gut microbiota could be sufficient to induce some phenotypical changes of obesity by FMT. The mice fed HFD or HFD plus $50 \mathrm{mg} / \mathrm{L}$ of fluoride in drinking water described in Animal protocol 2 was chosen as donor. Another 16 male C57BL/6J mice with 5 weeks of age were randomly divided into two groups $(\mathrm{n}=$ 8). At first two weeks, all mice were treated by Abx (containing $10 \mathrm{~g} / \mathrm{L}$ of vancomycin hydrochloride, 20 $\mathrm{g} / \mathrm{L}$ of neomycin sulfate, $20 \mathrm{~g} / \mathrm{L}$ of metronidazole and $20 \mathrm{~g} / \mathrm{L}$ of ampicillin sodium salt) by gavage with dosage of $200 \mathrm{~mL}$ for each mouse once daily according to the previous work to obtain the pseudogermfree mice $[49,68]$. At third week, FMT was carried out by transplanting the fecal slurry from donor mice to the pseudo-germfree mice until the end of the experiment as described previously [2,69]. Briefly, the fecal samples collected from donor mice (HFD and HFD-F groups) were immediately pooled and diluted in sterile saline containing $0.5 \mathrm{~g} / \mathrm{L}$ cysteine hydrochloride at proportion of $100 \mathrm{mg} / \mathrm{mL}$. Then, the fecal slurry was centrifuged at $1000 \mathrm{r} / \mathrm{min}$ for $1 \mathrm{~min}$ to obtain fecal supernatant. Each mouse received $200 \mathrm{~mL}$ of the fecal supernatant from HFD or HFD-F groups by gavage, respectively. The FMT continued for 8 weeks, and the mice were euthanized after fasting overnight at the end of the experiment.

Animal protocol 6. The purpose of this mice experiment was to investigate whether fluoride could exacerbate the obesity in the absence of gut microbiota. Sixteen male C57BL/6J mice with 5 weeks of age were randomly divided into two groups including HFD fed with Abx (HFD-Abx) group, and HFD fed with Abx plus $50 \mathrm{mg} / \mathrm{L}$ of fluoride in drinking water (HFD-Abx-F) group ( $\mathrm{n}=8$ per group). Abx was treated to mice by gavage once daily to clear gut microbiota according to the previous work with some modifications $[68,70]$. The intervention continued for 10 weeks, and the mice were euthanized after fasting overnight at the end of the experiment.

Animal protocol 7. The purpose of this mice experiment was to investigate whether fluoride could induce the obesity in ND mice. Sixteen male C57BL/6J mice with 5 weeks of age were randomly divided into two groups including ND group, and ND plus $50 \mathrm{mg} / \mathrm{L}$ of fluoride in drinking water (ND-F) group ( $\mathrm{n}=8$ per group). The intervention continued for 10 weeks, and the mice were euthanized after fasting overnight at the end of the experiment.

Animal protocol 8. The purpose of this mice experiment was to investigate whether E. ramosum could exacerbate the obesity in HFD mice (result obtained in animal experiment 2 and 5). Pseudo-germfree mice were obtained by treatment of Abx for first two weeks as described in Animal protocol 5. Then, pseudo-germfree mice were randomly divided into two groups including HFD fed with PBS and HFD fed with $E$. ramosum (HFD-ER) by intragastric gavage for two weeks. Each mouse received $0.2 \mathrm{~mL}$ of live $E$. ramosum $\left(10^{8}-10^{9} \mathrm{cfu} / \mathrm{mL}\right)$ daily. The intervention of $E$. ramosum continued for 2 weeks. After further 4 weeks, the mice were euthanized after fasting overnight at the end of the experiment. 
Animal protocol 9. The purpose of last mice experiment was to present a potential strategy to prevent fluoride-induced exacerbation of obesity in HFD mice by FBTPS. Sixteen male C57BL/6J mice with 5 weeks of age were randomly divided into two groups including HFD plus $50 \mathrm{mg} / \mathrm{L}$ of fluoride in drinking water (HFD-F) group, and HFD plus $50 \mathrm{mg} / \mathrm{L}$ of fluoride in drinking water and $400 \mathrm{mg} / \mathrm{kg} /$ day of FBTPS (HFD-F-TPS) group by intragastric gavage ( $\mathrm{n}=8$ per group). The mice in HFD-F group received same volume of water by intragastric gavage. The intervention continued for 8 weeks, and the mice were euthanized after fasting overnight at the end of the experiment.

\section{Caco-2 cell monolayer permeability assay in vitro}

The Caco-2 cell (HTB-37), obtained from American Type Culture Collection (ATCC, Manassas, VA, USA), was incubated in Dulbecco's modified Eagle's medium (DMEM) supplemented with $10 \%$ of fetal bovine serum, $1 \%$ of penicillin-streptomycin, $1 \%$ nonessential amino acids, and $1 \%$ L-glutamine at $37{ }^{\circ} \mathrm{Cunder} 5 \%$ of $\mathrm{CO}_{2}$ atmosphere. The cytotoxity of fluoride on Caco-2 cell was investigated by MTT assay. Briefly, Caco-2 cell suspension was plated in a 96-well plate $(200 \mathrm{~mL} /$ well $)$, and cultured for $24 \mathrm{~h}$. Then, the new medium containing various concentrations of fluoride $(0,6.25,12.5,25,50,100,200$ and $400 \mathrm{mg} / \mathrm{mL})$ were added to replace the old medium, and further incubated for $24 \mathrm{~h}$. After discarding the culture medium, MTT solution was added and incubated for a $4 \mathrm{~h}$. Then, MTT solution was removed and $200 \mathrm{~mL}$ of DMSO was added. The cytotoxicity was calculated using the absorbance (Abs) measured at $570 \mathrm{~nm}$. The effect of fluoride on the monolayer permeability was investigated according to the previous work [71, 72]. Caco-2 cell suspension was plated onto the polycarbonate membranes of Millicell inserts into a tween-well Transwell plate ( $0.4 \mathrm{~mm}$ pores; Corning, NY, USA) at a density of $1 \times 10^{5}$ cells per well. The cell medium in the apical and basolateral sides was renewed every two days at first 14 days and renewed daily at last 7 days. The transepithelial electrical resistance (TEER) was measured by a Millipore ERS-2 system (Millipore Crop., New Bedford, MA, USA). At 21th day, the wells with TEER values of more than $500 \Omega \mathrm{cm}^{2}$ were chosen for further experiments. After washing by PBS, $0.5 \mathrm{~mL}$ of cell medium (without fetal bovine serum) containing 0,10 and $50 \mathrm{mg} / \mathrm{L}$ of fluoride were added into the apical side of well in Transwell plate, and $1.5 \mathrm{~mL}$ of cell medium without fetal bovine serum were added into the basolateral sides of well in Transwell plate. After incubation at $37{ }^{\circ} \mathrm{C}$ for $12 \mathrm{~h}$, TEER values were measured again for evaluating the effect of fluoride on the monolayer permeability in vitro.

\section{The lipid metabolism assay in HepG2 cells in vitro}

HepG2 cells were purchased from National Collection of Authenticated Cell Cultures (Shanghai, China). HepG2 cells were incubated in DMEM supplemented with $10 \%$ of fetal bovine serum, and $1 \%$ of penicillinstreptomycin, at $37^{\circ} \mathrm{C}$ under $5 \%$ of $\mathrm{CO}_{2}$ atmosphere. The cytotoxity of fluoride on HepG2 cells was investigated by MTT assay as described for Caco-2 cell. The effect of fluoride on the lipid metabolism was evaluated in HepG2 cells in vitro as described previously with some modifications [73]. HepG2 cell suspension was plated in a 12-well plate with $1 \mathrm{~mL} /$ well, and cultured for $24 \mathrm{~h}$. Then, the serum-free medium, containing $100 \mathrm{mM}$ of palmitic acid, $200 \mathrm{mM}$ of oleic acid and different dosages of fluoride (0, 10 and $50 \mathrm{mg} / \mathrm{L}$, respectively), was added to replace the old medium, the cells were further incubated for 
$24 \mathrm{~h}$ to induce excess fat synthesis. After washed by PBS for three times, the cells were stained with Oil Red $\mathrm{O}$ solution to evaluate the level of intracellular fat droplets. Furthermore, the Oil Red $\mathrm{O}$ was extracted by $100 \%$ isopropyl alcohol, and the absorbance of the extracted isopropyl alcohol solution was measured at $492 \mathrm{~nm}$ to further quantify the intracellular lipid content. Moreover, the triglyceride (TG) and protein contents in HepG2 cells were measured by commercially available kits (Nanjing Jiancheng Bioengineering Institute, Nanjing, China).

\section{Cultivation of Erysipelatoclostridium ramosum}

E. ramosum CCUG35705 was purchased from Culture Collection University of Gothenburg (CCUG, Sweden). E. ramosum was cultured in brain heart infusion (BHI) medium at $37^{\circ} \mathrm{C}$ at an anaerobic workstation (HYQX-囚, Shanghai Yuejin Medical Instrument Co., LTD, Shanghai, China). After incubation for $12 \mathrm{~h}$, the E. ramosum was centrifuged at $4000 \mathrm{~g}$ for $5 \mathrm{~min}$, and resuspended in anaerobic PBS containing $10^{8}-10^{9} \mathrm{cfu} / \mathrm{mL}$ of $E$. ramosum. Then, the $E$. ramosum would be gavaged to mice immediately.

\section{Oral glucose tolerance test (OGTT)}

One week before the end of animal experiment, the mice were fasted overnight for OGTT. After measurement of blood glucose, the mice were immediately given an oral gavage glucose at a dosage of $1.5 \mathrm{~g} / \mathrm{kg}$ body weight. Then, the blood glucose was further measured after 15, 30, 60, 90 and $120 \mathrm{~min}$. All blood samples were collected from the tip of the tail vein, and the blood glucose was measured using a glucose meter (Sinocare Inc., Changsha, Hunan, China).

\section{Tissue sampling}

The animals were anesthetized with carbon dioxide, and blood, liver, colon, perirenal fat, mesentery fat, and epididymal fat tissues were collected. The blood sample was collected in sterile anticoagulation tube (BD Biosciences, USA). Then, $50 \mathrm{~mL}$ of blood was immediately taken out and stored at $-80^{\circ} \mathrm{C}$ for quantification of Bacterial DNA. The residue blood was centrifuged at $3000 \mathrm{r} / \mathrm{min}$ for $15 \mathrm{~min}$ at $4{ }^{\circ} \mathrm{C}$ to afford plasma. After weighing and taking picture, a part of the liver, colon, and epididymal fat tissues were fixed in $4 \%$ paraformaldehyde solution for histological analysis. Other tissues were stored at $-80{ }^{\circ} \mathrm{C}$ for further experiment.

\section{Biochemical analyses}

The contents of TG, total cholesterol (TC), low-density lipoprotein cholesterol (LDL-C) and in plasma and TG in liver were measured by commercially available kits (Nanjing Jiancheng Bioengineering Institute, Nanjing, China). The levels of tumor necrosis factor-a (TNF-a), interleukin-6 (IL-6) and IL-1b in plasma were determined using commercial ELISA kits (Neobioscience Technology Co, Ltd., Shenzhen, China). The lipopolysaccharide (LPS) in plasma was determined using commercial ELISA kit (Nanjing Jiancheng 
Bioengineering Institute, Nanjing, China). The insulin in plasma was determined using commercial ELISA kit (Mercodia AB, Uppsala, Sweden).

\section{Quantification of bacterial DNA in blood}

The quantification of bacterial DNA in blood were carried out according to previous work with some modifications [67]. The whole DNA in blood $(50 \mathrm{~mL})$ was extracted using a commercially available kit (TIANGEN BIOTECH (BEIJING) CO., LTD., Beijing, China). The concentrations of DNA were analyzed by Nanodrop 2000 (Thermo Fisher Scientific Inc., USA), and the bacterial DNA in blood was quantified by Femto $^{\text {TM }}$ Bacterial DNA Quantification Kit (ZYMO RESEARCH, USA).

\section{RNA extraction and Quantitative real-time PCR}

The total RNA of liver and colon was extracted by commercially available kits (YFXM0013, YIFEIXUE Biotechnology, Nanjing, China). After quantified by Nanodrop 2000 (Thermo Fisher Scientific Inc., USA), the RNA was reversed to cDNA by PrimeScript RT Master Mix (TaKaRa). The resulting cDNA was used for quantitative real-time reverse-transcription PCR (RT-qPCR) using SYBR Green Master Mix (A25742, ABI, USA) and QuantStudio 6 Flex (Thermo Fisher Scientific Inc.). The expression of mRNA was normalized to glyceraldehyde-3-phosphate dehydrogenase (GAPDH) by the method of $2^{-\Delta \Delta C t}$. Primers were obtained from Sangon Biotech (Shanghai, China), which was presented in Supplementary Table 2.

\section{Histology analysis and immunofluorescence assessment}

The histological analyses of liver, epididymal fat and colon were carried out according to the previous work $[14,38]$. After fixed in $4 \%$ paraformaldehyde solution, the liver, epididymal fat and colon tissues were embedded in paraffin, and stained with hematoxylin and eosin (H\&E). The liver tissue was also stained with Sirius Red. The protein expressions of ZO-1, Occludin, Claudin-1 and MUC1 in the colon tissue were also evaluated by immunofluorescence assessment as previously described [74]. After deparaffinized and rehydrated, the colon samples were incubated twice in xylene for 15 min each, dehydrated twice in pure ethanol for 5 min each, dehydrated in gradient ethanol of $85 \%$ and $75 \%$ ethanol for 5 min each, and washed in distilled water, respectively. The samples were dipped in $3 \%$ of bull serum albumin (BSA) for 30 min to block non-specific binding. The BSA solution was discarded, the primary antibody, including AntiZO-1 (ab221547), Anti-Occludin (ab216327), Anti-Claudin-1 (ab211737) and Anti-MUC1 (ab45167) (Abcam, Cambridge, UK), diluted in PBS solution was added and incubated at $4{ }^{\circ} \mathrm{C}$ overnight. After washed by PBS for three times, the sample was further incubated in secondary antibody solution (Jackson ImmunoResearch, Philadelphia, USA) for 50 min in dark condition at room temperature. The sample was washed by PBS three times and incubated with 4',6-diamidino-2-phenylindole (DAPI, Beyotime Biotechnology, Shanghai, China) solution in dark condition at room temperature. After washed by PBS for three times, the sample was added into the spontaneous fluorescence quenching reagent, incubated for $5 \mathrm{~min}$, and further washed in running tap water for $10 \mathrm{~min}$. Then, the samples were detected and collected by Fluorescent Microscopy. The mean density of immunofluorescence assessment was quantified by ImageJ software (1.8.0_172). 


\section{Gut microbiota analysis by $16 \mathrm{~S}$ rRNA amplicon sequencing}

The extraction of fecal genomic DNA in fecal samples was carried out using E.Z.N.A. ®Stool DNA Kit (D4015, Omega, Inc., USA). The 16S rRNA gene sequencing was performed by LC-Bio Technology Co., Ltd (Hangzhou, China). Briefly, the variable V3-V4 region of $16 \mathrm{~S}$ rDNA gene was amplified with the primers 341F CCTACGGGNGGCWGCAG, and 805R GACTACHVGGGTATCTAATCC by PCR using 32 amplification cycles. After detected using $2 \%$ of agarose gel electrophoresis, the target fragments were further purified by AMPure XT beads (Beckman Coulter Genomics, Danvers, MA, USA) and quantified by Qubit (Invitrogen, USA). Then, the resulting PCR product was loaded onto NovaSeq PE250 platform using a paired-end sequencing protocol $(2 \times 250 \mathrm{bp})$ according to the manufacturer's recommendations. The paired-end reads were identified by unique barcode of each sample. After cutting off the barcode and primer sequence, paired-end reads were merged by FLASH (v1.2.8). According to the fqtrim (v0.94), the highquality clean tags was obtained after the quality filtering of raw reads under specific filtering conditions. Vsearch software (v2.3.4) was used to filter chimeric sequences. Then, the feature table and feature sequence were obtained after dereplication by DADA2. The alpha diversity and beta diversity were analyzed using QIIME2. The sequence alignment of species was annotated by Blast based on databases of SILVA (Release 132, https://www.arb-silva.de/documentation/release-132/) and NT-16S. The linear discriminant analysis (LDA) effect size (LEfSe) was used to excavate the key gut microbiota in each group.

\section{Quantification of $E$. ramosum in fecal samples}

The quantification of E. ramosum in fecal samples was carried out according to the previous work [75]. Briefly, DNA in fecal samples was extracted by a commercially available kit (TIANGEN BIOTECH (BEIJING) CO., LTD., Beijing, China). The levels of DNA were quantified by Nanodrop 2000 (Thermo Fisher Scientific Inc., USA). Gene levels of E. ramosum were determined by RT-qPCR using SYBR Green Master Mix (A25742, ABI, USA) and QuantStudio 6 Flex (Thermo Fisher Scientific Inc.). $50 \mathrm{ng}$ of bacterial DNA was used to perform the RT-qPCR for each sample. The primer of $E$. ramosum used in this work was as fellow: FW: ACAATGGATGGTGCAGAGGG, RV: TCAACTCTCTCGTGGTGTGACG [61]. Higher CT values suggest lower levels of $E$. ramosum.

\section{Human cohort analyses}

The data of from the American Gut Project (AGP) was reanalyzed to gain insight into the relationship between the relative abundance of Erysipelotrichaceae and obesity according to the previous work [76, 77]. The subjects which contained fecal samples were chosen. The samples, who were treated with antibiotic, had diabetes or whose information such as body mass index (BMI), sex was missing, were removed. Furthermore, the subjects whose BMI were more than 50 or less than $18.5 \mathrm{~kg} / \mathrm{m}^{2}$ were also excluded. Finally, a total of 10,376 individuals aged from 20 to 99 years, including 6546 normal, 2869 overweight and 961 obesity subjects, were obtained in this work. The sequence read files of these subjects were obtained from European Bioinformatics Institute (EBI, PRJEB11419)[33]. The sequences 
were analyzed using pipeline as previously described [78] using Rstudio (1.4.1106), vsearch (v2.15.2) and usearch (v10.0.240). The ASV table was obtained by usearch, and the sequence alignment of species was annotated by vsearch according to the databases of silva_16s_v123. The subjects with less than 5000 clean reads were removed. After merging all annotation of ASVs to taxonomy tables, the relative abundance of Erysipelotrichaceae was obtained for each subject.

\section{Statistical analysis.}

The results were expressed as means \pm SEM. Statistical significance was carried out by one-way analysis of variance (ANOVA) with Tukey test for multiple-group comparisons. Difference in two groups was calculated using the Mann-Whitney test or Kruskal-Wallis test. The correlations between relative abundances of Erysipelotrichaceae and BMI were analyzed by Pearson correlation. Statistical analysis was carried out by SPSS 22 software (IBM). A value of $p<.05$ was considered to be significant.

\section{Results}

\section{Fluoride exacerbates the obesity in HFD mice}

To determine the effects of fluoride on obesity, the fluoride was added into the drinking water at a concentration of $50 \mathrm{mg} / \mathrm{L}$ to HFD fed mice (Fig. S1A). The dosage in this work was chosen according to the previous work [21]. As expected, HFD could significantly induce the features of obesity compared with the ND group (Fig. S1). It was observed that fluoride remarkably exacerbated the obesity in HFD mice over a 10-week period, including increasing body weight (Fig. S1B-C), accumulation of fat tissues (Fig. S1D-H), increased plasma levels of triglyceride (TG), total cholesterol (TC), and glucose (Fig. S1I-K). Furthermore, fluoride could increase liver weight, hepatic TG and result in obvious liver steatosis (Fig. S1L-N). As shown in Fig. S10, fluoride exposure did not affect the food intake $(p=0.985)$, suggesting that exacerbation of the obesity by fluoride was not related to the change of energy intake. The reduced colon length and histological damage in colon tissue were observed (Fig. S2A-C). Furthermore, the protein expression of Claudin-1 and ZO-1 in colon was down-regulated by fluoride compared with those of the ND and HFD groups (Fig. S2D-G). Thus, the exacerbation of obesity by fluoride might be related to the gut health. In addition, a separate replicate experiment was carried out to further confirm the effect of fluoride on the obesity in HFD mice, and similar results were observed as showed in Fig. 1, that fluoride could exacerbate the obesity in HFD mice. The fluoride could also deteriorate the glucose tolerance by significant increase of glucose levels in 0 and $15 \mathrm{~min}$, and increase of aera under the curve (AUC) during the oral glucose tolerance test (OGTT) as shown in Fig. 1K-L. Furthermore, the liver injury and slight liver fibrosis were observed after exposure of fluoride according to the high level of plasma alanine transaminase (ALT) and Sirius red in liver tissue (Fig. 1P-Q). Thus, fluoride treatment could exacerbate the detrimental effect of HFD on features of obesity.

Fluoride drives the intestinal barrier permeability and deteriorates the inflammation in HFD mice 
As described above, the detrimental effect of fluoride might be related to the gut health. Thus, the effect of fluoride on the intestinal barrier permeability was further investigated in the Experiment 2. Similarly, fluoride could reduce the colon length (Fig. 2A-B), lead to histological damage and inflammation in colon tissue (Fig. 2C-D), and decrease the mRNA and protein expressions of Claudin-1, MUC1 and ZO-1 in colon evaluated by RT-qPCR and immunofluorescence assay (Fig. 2E-N). The intestinal barrier permeability would lead to leaky gut, and some of gut microbiota, along with toxins such as LPS, might leak into the bloodstream, which was regarded as an important mechanism for metabolic diseases [29]. In this work, the level of bacteria in blood and plasma LPS concentration were significantly increased in the HFD-F group compared with those in the ND or HFD groups (Fig. 20-P). The effect of fluoride on intestinal barrier function was further evaluated in vivo using a fluorescein isothiocyanate (FITC)-dextran-based intestinal permeability assay (Fig. 2Q) and in vitro using Caco-2 cell model (Fig. S3). It was found that plasma FITC-dextran level in the HFD-F group was significantly higher than that in the HFD group ( $p$ $=0.038$ ). Moreover, fluoride derived Caco-2 cell permeability in vitro evaluated by decreasing transepithelial electrical resistance (TEER). Thus, the experiments in vitro and in vivo both demonstrated that fluoride could drive the intestinal barrier permeability. After leaking into the bloodstream, the bacteria and LPS could activate the TLR4, and thereby leading to metabolic inflammation and obesity [15]. Thus, the plasma levels of tumor necrosis factor-a (TNF-a), interleukin-6 (IL-6) and IL-1b, and relative mRNA expression levels of Tnf-a, II- $6, \|-1 b$ in liver were evaluated, and the results showed that fluoride could deteriorate the inflammation in the HFD mice (Fig. S4A-F). Furthermore, fluoride could upregulate the mRNA expression levels of Myd88 and T/r4 in liver, suggesting that the exacerbation of obesity by fluoride might depend on an activation of TLR4 mechanism.

\section{Fluoride exacerbates the obesity in HFD mice through a TLR4-dependent mechanism}

As described above, the exacerbation of obesity by fluoride might be related to an activation of TLR4 mechanism. Thus, T/r4 knockout ( $\mathrm{TIr}^{-/}{ }^{-}$) mice and wild-type (WT) mice were used to further demonstrate the role of TLR4 in the potential mechanisms (Fig. 3A). As expected, fluoride could significantly exacerbate the obesity in WT mice (Fig. 3), especially deteriorating the glucose tolerance (Fig. 3K-I), and inducing lipid accumulation and steatosis in liver tissues (Fig. 3M-R). Furthermore, the inflammation, reflecting as increasing levels of TNF-a, IL-6, and IL-1b in plasma, was observed in WT mice (Fig. S5A-F). However, the exacerbation of obesity and inflammation by fluoride was blocked after knockout of T/r4. The results showed that detrimental effect of fluoride on HFD induced obesity was partially dependent on TLR4 mechanism.

\section{Fluoride disturbs the gut microbiota in HFD mice}

The effect of fluoride on the lipid metabolism was investigated using an oleic acid/palmitic acid (OA/PA) induced HepG2 cell model (Fig. S6). It was found that fluoride showed limited effect on lipid accumulation evaluated by level of $\mathrm{TG}$ and oil red $\mathrm{O}$ staining. Thus, fluoride might not directly lead to exacerbation of the obesity in HFD mice. Recently, evidence is accumulating that dysbiosis of gut microbiota is involved in the development of obesity $[7,30]$. Moreover, it has been widely reported that 
fluoride could affect the gut microbiota [31,32]. Thus, we suspected that fluoride induced changes of gut microbiota might contribute to the exacerbation of the obesity. As shown in Fig. S7, the fluoride showed no effect on a-diversity of gut microbiota evaluated by observed_otus, Chao1, Shannon, and Simpson indexes, while b-diversity including principal component analysis (PCA) and Principal coordinates analysis (PCOA) showed that HFD could significantly change the structure of gut microbiota, and fluoride showed week influence on the composition of gut microbiota (Fig. 4A-B). At the phylum level, HFD induced a decreased relative abundance of Bacteroidetes, and an increased relative abundance of Firmicutes, thereby significantly enhance the ratio of Firmicutes to Bacteroidetes (Fig. 4C-F), which was in accordance with the previous works [1,9]. Whereas, fluoride showed limited effects on the relative abundances of Bacteroidetes and Firmicutes and the ratio of Firmicutes to Bacteroidetes. Then, the linear discriminant analysis effect size (LEfSe) analysis was used to excavate the key gut microbiota changed by fluoride (Fig. 4G-H). It was found that Erysipelotrichaceae at the family level was significantly higher than those in the ND and HFD groups. As shown in Fig. 4I, the HFD increased the relative abundance of Erysipelotrichaceae, whereas, fluoride could further stimulate the proliferation of Erysipelotrichaceae in the HFD fed mice. Thus, Erysipelotrichaceae at the family level might be the key gut microbiota in the HFD-F group contributing to the exacerbation of obesity.

To further evaluate the relationship between Erysipelotrichaceae, the key candidate gut microbiota, and obesity in humans, AGP was applied to demonstrate our prediction [33]. Thus, the association between Erysipelotrichaceae and BMI based on the database of AGP was analyzed using Pearson correlation (Fig. 5), and the result showed that the relative abundance of Erysipelotrichaceae showed significantly positive relation to $\mathrm{BMI}(\mathrm{n}=10376, p<0.0001)$. Furthermore, the relative abundance of Erysipelotrichaceae in overweight and obesity objects was significantly higher than that in normal subjects $(p<0.01)$. Thus, the result further supported our prediction resulting from our analyses in the mice experiment, that Erysipelotrichaceae might be served as the microbial signature of dysbiosis induced by fluoride in HFD mice. The Erysipelatoclostridium at the genus level belonging to Erysipelotrichaceae increased significantly after fluoride exposure compared with that in the ND or HFD groups (Fig. 4J). Then, RT-qPCR and species-specific primers were used to identify the specific gut microbiota at the species level belonging to Erysipelatoclostridium, it was found that Erysipelatoclostridium ramosum significantly increased after exposure of fluoride, which might be the potential key bacteria for fluoride intervention (Fig. 4K).

\section{Fecal microbiota transplant (FMT) is sufficient to induce some phenotypical changes caused by fluoride in HFD mice}

FMT is an effective way to validate the role of the gut microbiota in disease, and further identify the specific bacteria responsible for development of disease [34]. To evaluate whether gut microbiota from fluoride-induced mice could lead to obesity, FMT from either the HFD group or HFD-F group mice to pseudo-germfree mice induced by antibiotic cocktail (Abx) was carried out (Fig. 6A). It was found that FMT from fluoride-induced mice was sufficient to induce some phenotypical changes, mainly including increasing body weight, accumulation of fat tissues, plasma levels of low-density lipoprotein cholesterol 
(LDL-C), ALT, liver damage and deteriorating the glucose tolerance (Fig. 6B-O). Although the fluorideinduced gut microbiota could not change the colon length, it impaired gut barrier function and promote the inflammation (Fig. S8). At the same time, the gut microbiota in recipient mice was analyzed (Fig. 7). According to the results of PCA and PCoA, the structure of gut microbiota from recipient mice receiving FMT from HFD or HFD-F group was different (Fig. 7A-B, $p=0.004$, and $p=0.001$ for PCA and PCoA, respectively). The relative abundances of Bacteroidetes and Firmicutes, and the ratio of Firmicutes to Bacteroidetes showed no significant difference between different groups (Fig. 7C-F). The LEfSe analysis showed that Erysipelotrichaceae and Erysipelatoclostridium in recipient mice receiving FMT from the HFD-F group were higher (Fig. 7G-H), which was further confirmed by statistical analysis (Fig. 7I-J). Thus, the fluoride-induced key gut microbiota could colonize in pseudo-germfree mice by FMT, and thereby exacerbated the development of obesity in HFD mice.

\section{The exacerbation of the obesity by fluoride is blocked after depletion of the gut microbiota by Abx}

As described above, the exacerbation of the obesity by fluoride might be related to the gut microbiota. Thus, the effect of fluoride on the obesity in HFD mice after depletion of the gut microbiota by Abx was performed to investigate whether fluoride could exacerbate the obesity in the absence of gut microbiota (Fig. 8A). The level of key gut microbiota was measured by RT-qPCR at the end of experiment, and the results showed that CT values of E. ramosum was more than 34, which showed that the level of $E$. ramosum in fecal samples was greatly reduced after depletion of the gut microbiota by Abx (Fig. S9). As expected, no remarkably difference was observed in phenotypical changes of obesity after fluoride exposure in the absence of gut microbiota (Fig. 7B-O). Furthermore, fluoride failed to induce the inflammation after depletion of the gut microbiota (Fig. S10). Thus, the detrimental influence of fluoride on the HFD-induced obesity was blocked after depletion of the gut microbiota, suggesting that the exacerbation of the obesity by fluoride was involved in a microbiota-dependent manner.

\section{Fluoride fails to induce the obesity in ND mice}

The present result showed that fluoride could exacerbate the obesity in HFD fed mice. However, whether fluoride could induce the metabolic disease in ND fed mice is still unknown, which was evaluated in the present study (Fig. S11A). Except significantly decreasing the plasma level of TC, fluoride showed limited effect on the phenotypical changes of obesity (Fig. S11B-O), indicating that fluoride failed to induce the obesity in ND mice. In addition, the gut microbiota was analyzed using 16S rRNA gene sequencing (Fig. S12). The results showed that fluoride could not change the structure or composition of gut microbiota evaluated by a-diversity including observed_otus, Chao1, Shannon, Simpson indexes, b-diversity including PCA and PCoA, and bacterial taxonomic profiling at the phylum level including the relative abundances of Firmicutes, Bacteroidetes, and the ratio of Firmicutes to Bacteroidetes $(p>0.05$, Fig. S12A-J).

Furthermore, the relative abundances of Erysipelotrichaceae and Erysipelatoclostridium showed no response after fluoride exposure ( $p>0.05$, Fig. S12K-L), highlighted the key role of gut microbiota and Erysipelotrichaceae in the detrimental effect of fluoride on the obesity.

\section{E. ramosum aggravates the obesity in HFD mice}


As described above, Erysipelotrichaceae might be the key bacterium for aggravation of obesity by fluoride. Thereinto, E. ramosum, formerly Clostridium ramosum that has been transferred to the new genus Erysipelatoclostridium in the family Erysipelotrichaceae [35], was identified as one of the potential key bacteria increased by fluoride. Thus, the effect of $E$. ramosum on the obesity was performed to investigate whether $E$. ramosum could exacerbate the obesity in HFD mice (Fig. 9A). After intervention with $E$. ramosum, the body weight, epididymal fat, plasma levels of TC and LDL-C were all significantly increased (Fig. 9B-J). The glucose tolerance, liver fibrosis and steatosis were also deteriorated by $E$. ramosum (Fig. 9K-0). Furthermore, E. ramosum aggravated the inflammation in HFD mice (Fig. S13). Thus, E. ramosum could aggravate the obesity in HFD mice, which might be regarded as a microbial signature of dysbiosis of gut microbiota induced by fluoride.

\section{Fuzhuan brick tea polysaccharides (FBTPS) abolish the fluoride-induced obesity in HFD mice}

In recent years, the prebiotics have received increasing attention due to the perfect gut microbiota management with selective promotion of beneficial gut microbiota and inhibition of harmful bacteria [36, 37]. Thus, it was expected to explore a novel strategy for prevention of fluoride-induced obesity based on the modulation of gut microbiota. In our previous work, FBTPS could restore the increases in relative abundances of Erysipelotrichaceae induced by HFD [38]. In the present study, the effect of FBTPS on fluoride induced obesity was investigated (Fig. 9A). Except epididymal and perirenal fat tissues, FBTPS could significantly relieve all the phenotypical changes of obesity induced by fluoride (Fig. 10B-P). Furthermore, FBTPS could abolish the fluoride-induced inflammation in HFD fed mice (Fig. S14). Thus, the intervention of FBTPS might be served as promising candidate for prevention of fluoride-induced obesity.

\section{Discussion}

Due to industrial pollution and geological origin, fluoride is widespread in our environment, drinking water and various foods [39]. Furthermore, fluoride is a widely used additive in mouthwashes and toothpastes to prevent tooth decay and protect our tooth [40]. Thus, the toxicity of fluoride has attracted increasing attention in recent years [22]. Thereinto, the fluoride-associated hepatotoxicity, impairing glucose tolerance and lipid metabolism disorder have been previously reported [27, 41, 42], however, the understanding mechanisms of fluoride-induced obesity are still not comprehensive, and the role of gut microbiota in the exacerbation of the obesity is ignored. In this work, the potential adverse effect of fluoride on the obesity was investigated detailly, furthermore, the potential mechanism was proposed, that fluoride exacerbated the obesity in HFD mice via disturbing gut microbiota and driving intestinal barrier permeability.

Firstly, we confirmed that fluoride with a dosage of $50 \mathrm{mg} / \mathrm{L}$ in the drinking water could significantly exacerbate the obesity in mice fed with HFD but not ND according to two separate replicate mice experiments. More specifically, it was found that fluoride further increased body weight, accumulation of fat, liver damage in HFD induced obese mice, whereas no phenotypical change of obesity was observed 
in ND fed mice after exposure of fluoride with same dosage of fluoride for 10 weeks. It has been reported that fluoride affected the lipid metabolism in a diet-dependent pattern [43]. Fluoride induced steatosis and dyslipidemia in animals fed hypercaloric diets, whereas, no change was observed in normocaloric-fed rats [28]. Furthermore, fluoride with the same dosage could enhance glucose homeostasis along with a normocaloric diet, which might show beneficial effect on diabetes [44]. Likewise, fluoride could decrease the level of plasma level of TC in ND fed mice in this work. Thus, the beneficial or detrimental fluoride to health also depended on the diets. In another similar work, foodborne titanium dioxide nanoparticles showed a stronger detrimental effect on colonic inflammation in obese mice than non-obese mice [45].

The integrity of the epithelial barrier plays an important role in defensing against the invasion of microorganisms and preventing leakages of microbial products such as LPS into the bloodstream [46]. After intestinal barrier dysfunction and dysbiosis, the microorganisms and LPS in bloodstream released from colon could stimulate TLR4 receptor, and thereby induce inflammation and metabolic diseases [29, 47]. The dietary habits, such as HFD, food additives, probiotics, could affect the intestinal barrier [48]. Recently, some food contaminants, additives or food-derived mycotoxins could directly or indirectly impair the intestinal integrity, and thereby promoting the colitis, liver inflammation or metabolic syndrome $[18,49,50]$. The adverse effect of fluoride on intestinal barrier has been reported [51, 52]. In this work, it was found that fluoride could drive the intestinal barrier permeability in mice model, which was further verified by in vitro experiment using Caco-2 cells and in vivo experiment using FITC-dextran-based intestinal permeability assay. Moreover, the microorganisms in blood and LPS in plasma were enhanced by exposure of fluoride. Thus, we hypothesize that microorganisms and LPS in bloodstream could activate TLR4 to cause low-grade inflammation, and thereby exacerbate the obesity. After confirming the upregulation of T/r4 and Myd88 at mRNA level along with the low-grade inflammation in liver, TIr4 ${ }^{-/}$mice and WT mice were applied to demonstrate our hypothesis. Fluoride could still exacerbate the obesity in WT mice, whereas, exacerbation of inflammation and obesity by fluoride was blocked in $\mathrm{Tlr}^{-/-}$mice. Together, these results suggest that fluoride exacerbates the obesity in HFD mice via driving the intestinal barrier permeability and activating TLR4 signaling.

In the present work, the limited effect of fluoride on the lipid accumulation was observed using HepG2 cell model in vitro. Fluoride may not directly affect the lipid metabolism in HFD fed mice. Besides the intestinal barrier permeability as described above, accumulating evidence has demonstrated that the gut microbiota dysbiosis is associated with pathologic conditions of obesity and obesity-related complications [7, 30]. Furthermore, the changes of gut microbiota induced by fluoride have been reported to be related to its adverse effects, such as neurodevelopmental and cardiovascular impacts [32, 53]. Thus, we investigated the potential involvement of the gut microbiota in mediating fluoride-induced obesity. Firstly, the results of FMT experiment showed that fluoride-induced gut microbiota was sufficient to induce some phenotypical changes, suggesting the gut microbiota was involved in fluoride-mediated obesity. Furthermore, the exacerbation of obesity by fluoride was completely abrogated in mice after depletion of the gut microbiota by Abx, which highlighted that fluoride perturbed host-microbiota homeostasis rather than directly triggering host obesity. 
Then, 16S rRNA gene sequencing was applied to analyze the changes of gut microbiota after fluoride exposure, it was found that Erysipelotrichaceae at the family level was significantly increased, which might be responsible for exacerbation of the obesity by fluoride. Erysipelotrichaceae could colonize in pseudo-germfree mice by FMT, which might contribute to the obesity in recipient mice. The potential role of Erysipelotrichaceae in inflammation and metabolic disorders have been widely reported [54, 55], which was summarized by Kaakoush [56]. Importantly, the HFD could increase the abundance of Erysipelotrichaceae [38], then fluoride could further promote the proliferation of Erysipelotrichaceae, thereby exacerbated the HFD-induced obesity. Interestingly, fluoride failed to promote the proliferation of Erysipelotrichaceae in the absence of HFD, which might explain why fluoride could not induce obesity in ND mice. In a similar work, fluoride also showed limited effect on gut microbial communities in mice fed on standard mouse chow [57].

Based on the data from database of AGP, the relative abundance of Erysipelotrichaceae showed significantly positive correlation with BMI, further verified the key role of Erysipelotrichaceae in development of obesity. E. ramosum belonged to Erysipelotrichaceae was identified as one of potential key bacteria contributing to detrimental effect of fluoride on obesity. E. ramosum was associated with systemic low-grade inflammation, metabolic parameters and corpulence traits $[58,59]$. Furthermore, the promotion of HFD-induced obesity in mice has been reported after administration of E. ramosum [60, 61]. In this work, E. ramosum could exacerbate the inflammation and obesity in HFD mice. Moreover, $E$. ramosum showed a positively association with many diseases, such as COVID-19 disease, colorectal cancer $[62,63]$, which has been regarded as a microbial signature of microbiota dysbiosis. Thus, we would like to emphasize that $E$. ramosum was one of key gut microbiota contributing to detrimental effect of fluoride on obesity, which could be considered as a microbial signature of fluoride-induced microbiota dysbiosis. However, whether other bacteria belonged to Erysipelotrichaceae being responsible for exacerbation of the obesity by fluoride is still one of the open-ended questions of our study.

Prebiotics, one microbiota-management tool, could inhibit the harmful pathogen, and stimulate the proliferations of beneficial bacteria, thereby improve human health [36, 37]. Thus, the development of microbiota-directed dietary fibers is expected as a novel strategy to prevent the gut microbiota-related diseases $[64,65]$. As described above, the exacerbation of the obesity in HFD mice might depend on the bloom of Erysipelotrichaceae after exposure of fluoride. Thus, it was expected to explore a novel dietary fiber to inhibit the proliferation of Erysipelotrichaceae, thereby prevent the fluoride-induced exacerbation of obesity. In our previous work, FBTPS treatment could prevent the proliferation of Erysipelotrichaceae in HFD induced obese mice [38]. As expected, FBTPS could abolish the fluoride-induced obesity in HFD mice in this work. Some functional foods also showed to reduce the fluoride-induced detrimental effects. For example, rutin could protect fluoride induced dyslipidemia, blood toxicity and cardiotoxicity in rats [66]. Thus, we open up exciting therapeutic avenues for prevention of fluoride-induced obesity, which used FBTPS as a potential candidate for modulation of gut microbiota.

In conclusion, we found that fluoride, widely consumed by humans, could exacerbate the obesity in HFD fed mice, while the fluoride alone did not show detrimental effect on the obesity in ND fed mice. 
Furthermore, we provided the first evidence of a comprehensive cross-talk mechanism between fluoride and obesity in HFD fed mice, which is mediated by gut microbiota and intestinal barrier. Another finding is that Erysipelatoclostridium ramosum was identified as a crucial mediator of fluoride induced obesity, which could be explored as potential target for prevention and treatment of obesity with exciting translational value. Finally, FBTPS is suggested as a potential candidate to prevent fluoride induced obesity.

\section{Declarations}

\section{Acknowledgments}

We would like to thank all the technicians in the Experimental Animal Center of Nanjing Agricultural University for assistance with animal experiment.

\section{Funding}

The study was supported by a grant-in-aid from the Key Technology R\&D Program of Jiangsu Province (BE2020341), the National Key Research and Development Program of China (2018YFC1604404), the Fundamental Research Funds for the Central Universities (KJQN202154), and a project funded by the Priority Academic Program Development of Jiangsu Higher Education Institutions.

\section{Author contributions}

X.Z. and Z.L. designed and supervised experiments. G.C., Y.P., W.D., W.X., Z.X., D.C., and W.Z. performed the experiments. G.C. and Y.H. analyzed the data. G.C. drafted the first versions. X.Z., M.X., Z.D., H.C., X.F., X.K., T.Y., C.C. and Y.S. discussed and revised the manuscript.

\section{Data availability}

All data needed to evaluate the conclusions in the paper are present in the paper and the Supplementary Materials. The raw sequence data generated in this work were deposited into the Genome Sequence Archive in the BIG Data Center Chinese Academy of Sciences (https://ngdc.cncb.ac.cn/gsa/) with BioProject number of PRJCA006688 under accession codes of CRA005089. Additional data related to this paper may be requested from the corresponding authors.

\section{Competing interests}

The authors declare no competing interests

\section{Ethics approval and consent to participate}

All animal experiment protocols were approved by the institutional Animal Ethics Committee of Experimental Animal Center of Nanjing Agricultural University. 


\section{References}

1. Chen G, Xie M, Dai Z, Wan P, Ye H, Zeng X, et al. Kudingcha and Fuzhuan Brick Tea Prevent Obesity and Modulate Gut Microbiota in High-Fat Diet Fed Mice. Mol Nutr Food Res. 2018;62(6):1700485.

2. Choi BSY, Daniel N, Houde VP, Ouellette A, Marcotte B, Varin TV, et al. Feeding diversified protein sources exacerbates hepatic insulin resistance via increased gut microbial branched-chain fatty acids and mTORC1 signaling in obese mice. Nat Commun. 2021;12(1):3377.

3. Du J, Zhang P, Luo J, Shen L, Zhang S, Gu H, et al. Dietary betaine prevents obesity through gut microbiota-drived microRNA-378a family. Gut Microbes. 2021;13(1):1862612.

4. López-Almela I, Romaní-Pérez M, Bullich-Vilarrubias C, Benítez-Páez A, Gómez Del Pulgar EM, Francés R, et al. Bacteroides uniformis combined with fiber amplifies metabolic and immune benefits in obese mice. Gut Microbes. 2021;13(1):1865706.

5. Pan X, Wang L, Pan A. Epidemiology and determinants of obesity in China. The Lancet Diabetes Endocrinol. 2021;9(6):373-92.

6. Rosenbaum M, Knight R, Leibel RL. The gut microbiota in human energy homeostasis and obesity. Trends Endocrinol. Metab. 2015;26(9):493-501.

7. Canfora EE, Meex RCR, Venema K, Blaak EE. Gut microbial metabolites in obesity, NAFLD and T2DM. Nat Rev Endocrinol. 2019;15(5):261-73.

8. Gimeno RE, Briere DA, Seeley RJ. Leveraging the Gut to Treat Metabolic Disease. Cell Metab. 2020;31(4):679-98.

9. Zeng S, Li S, Xiao P, Cai Y, Chu C, Chen B, et al. Citrus polymethoxyflavones attenuate metabolic syndrome by regulating gut microbiome and amino acid metabolism. Sci Adv. 2020;6(1):x6208.

10. Zhao L, Zhang F, Ding $X$, Wu G, Lam YY, Wang $X$, et al. Gut bacteria selectively promoted by dietary fibers alleviate type 2 diabetes. Science. 2018;359(6380):1151-6.

11. Zhao M, Jiang Z, Cai H, Li Y, Mo Q, Deng L, et al. Modulation of the Gut Microbiota during High-Dose Glycerol Monolaurate-Mediated Amelioration of Obesity in Mice Fed a High-Fat Diet. mBio;11(2):e120-90.

12. Gao X, Chang S, Liu S, Peng L, Xie J, Dong W, et al. Correlations between a-Linolenic Acid-Improved Multitissue Homeostasis and Gut Microbiota in Mice Fed a High-Fat Diet. Msystems. 2020;5(6):e32091.

13. Xiao L, Sonne SB, Feng Q, Chen N, Xia Z, Li X, et al. High-fat feeding rather than obesity drives taxonomical and functional changes in the gut microbiota in mice. Microbiome. 2017;5(1):43.

14. Zhang X, Coker OO, Chu ES, Fu K, Lau HCH, Wang Y, et al. Dietary cholesterol drives fatty liverassociated liver cancer by modulating gut microbiota and metabolites. Gut. 2021;70(4):761-774.

15. Chang C, Lin C, Lu C, Martel J, Ko Y, Ojcius DM, et al. Ganoderma lucidum reduces obesity in mice by modulating the composition of the gut microbiota. Nat Commun. 2015;6(1):7489.

16. Fabbiano S, Suárez-Zamorano N, Chevalier C, Lazarević V, Kieser S, Rigo D, et al. Functional Gut Microbiota Remodeling Contributes to the Caloric Restriction-Induced Metabolic Improvements. Cell 
Metab. 2018;28(6):907-21.

17. Suez J, Korem T, Zeevi D, Zilberman-Schapira G, Thaiss CA, Maza O, et al. Artificial sweeteners induce glucose intolerance by altering the gut microbiota. Nature. 2014;514(7521):181-6.

18. Chassaing B, Koren O, Goodrich JK, Poole AC, Srinivasan S, Ley RE, et al. Dietary emulsifiers impact the mouse gut microbiota promoting colitis and metabolic syndrome. Nature. 2015;519(7541):92-96.

19. He Z, Chen L, Catalan-Dibene J, Bongers G, Faith JJ, Suebsuwong C, et al. Food colorants metabolized by commensal bacteria promote colitis in mice with dysregulated expression of interleukin-23. Cell Metab. 2021;33(7):1358-1371.

20. Ayoob S, Gupta AK. Fluoride in Drinking Water: A Review on the Status and Stress Effects. Crit Rev Environ Sci Technol. 2006;36(6):433-87.

21. Guth S, Hüser S, Roth A, Degen G, Diel P, Edlund K, et al. Toxicity of fluoride: critical evaluation of evidence for human developmental neurotoxicity in epidemiological studies, animal experiments and in vitro analyses. Arch Toxicol. 2020;94(5):1375-415.

22. Kabir H, Gupta AK, Tripathy S. Fluoride and human health: Systematic appraisal of sources, exposures, metabolism, and toxicity. Crit Rev Environ Sci Technol. 2020;50(11):1116-93.

23. Jagtap S, Yenkie MK, Labhsetwar N, Rayalu S. Fluoride in Drinking Water and Defluoridation of Water. Chem Rev. 2012;112(4):2454-66.

24. Rizzu M, Tanda A, Cappai C, Roggero PP, Seddaiu G. Impacts of soil and water fluoride contamination on the safety and productivity of food and feed crops: A systematic review. Sci Total Environ. 2021;787:147650.

25. Peng C, Cai H, Zhu X, Li D, Yang Y, Hou R, et al. Analysis of Naturally Occurring Fluoride in Commercial Teas and Estimation of Its Daily Intake through Tea Consumption. J Food Sci. 2016;81(1):H235-9.

26. Johnston NR, Strobel SA. Principles of fluoride toxicity and the cellular response: a review. Arch Toxicol. 2020;94(4):1051-69.

27. Liu L, Wang M, Li Y, Liu H, Hou C, Zeng Q, et al. Low-to-moderate fluoride exposure in relation to overweight and obesity among school-age children in China. Ecotox Environ Safe. 2019;183:109558.

28. Pereira HABS, Dionizio AS, Fernandes MS, Araujo TT, Cestari TM, Buzalaf CP, et al. Fluoride Intensifies Hypercaloric Diet-Induced ER Oxidative Stress and Alters Lipid Metabolism. PLoS One. 2016;11(6):e158121.

29. Camilleri M. Leaky gut: mechanisms, measurement and clinical implications in humans. Gut. 2019;68(8):1516-1526.

30. Fan Y, Pedersen O. Gut microbiota in human metabolic health and disease. Nat Rev Microbiol. 2021;19(1):55-71.

31. Chen G, Hu P, Xu Z, Peng C, Wang Y, Wan X, et al. The beneficial or detrimental fluoride to gut microbiota depends on its dosages. Ecotox Environ Safe. 2021;209:111732. 
32. Qiu Y, Chen X, Yan X, Wang J, Yu G, Ma W, et al. Gut microbiota perturbations and neurodevelopmental impacts in offspring rats concurrently exposure to inorganic arsenic and fluoride. Environ Int. 2020;140:105763.

33. Mcdonald D, Hyde E, Debelius JW, Morton JT, Gonzalez A, Ackermann G, et al. American Gut: an Open Platform for Citizen Science Microbiome Research. Msystems. 2018;3(3):e18-31.

34. Giles EM, D Adamo GL, Forster SC. The future of faecal transplants. Nat Rev Microbiol. 2019;17(12):719.

35. Yutin N, Galperin MY. A genomic update on clostridial phylogeny: Gram-negative spore formers and other misplaced clostridia. Environ Microbiol. 2013;15(10):2631-2641.

36. Cunningham M, Azcarate-Peril MA, Barnard A, Benoit V, Grimaldi R, Guyonnet D, et al. Shaping the Future of Probiotics and Prebiotics. Trends Microbiol. 2021;29(8):667-85.

37. Sanders ME, Merenstein DJ, Reid G, Gibson GR, Rastall RA. Probiotics and prebiotics in intestinal health and disease: from biology to the clinic. Nat Rev Gastro Hepat. 2019;16(10):605-16.

38. Chen G, Xie M, Wan P, Chen D, Dai Z, Ye H, et al. Fuzhuan Brick Tea Polysaccharides Attenuate Metabolic Syndrome in High-Fat Diet Induced Mice in Association with Modulation in the Gut Microbiota. J Agr Food Chem. 2018;66(11):2783-95.

39. Xi J, Zhang L, Peng C, Zhou J, Peng Y, Xu L, et al. Flavor augmentations affect fluoride bioavailability from brewed dark tea. LWT-Food Sci Technol. 2019;109:270-5.

40. Ouyang Z, Yang B, Yi J, Zhu S, Lu S, Liu Y, et al. Exposure to Fluoride induces apoptosis in liver of ducks by regulating Cyt-C/Caspase 3/9 signaling pathway. Ecotox Environ Safe. 2021;224:112662.

41. García-Montalvo EA, Reyes-Pérez H, Del Razo LM. Fluoride exposure impairs glucose tolerance via decreased insulin expression and oxidative stress. Toxicology. 2009;263(2):75-83.

42. Yu Y, Zhou B, Yang Y, Guo C, Zhao J, Wang H. Estrogen Deficiency Aggravates Fluoride-Induced Liver Damage and Lipid Metabolism Disorder in Rats. Biol Trace Elem Res. 2021. https://doi.org/10.1007/s12011-021-02857-1

43. Dionizio A, Pereira HABS, Araujo TT, Sabino-Arias IT, Fernandes MS, Oliveira KA, et al. Effect of Duration of Exposure to Fluoride and Type of Diet on Lipid Parameters and De Novo Lipogenesis. Biol Trace Elem Res. 2019;190(1):157-71.

44. Lobo JGVM, Leite AL, Pereira HABS, Fernandes MS, Peres-Buzalaf C, Sumida DH, et al. Low-Level Fluoride Exposure Increases Insulin Sensitivity in Experimental Diabetes. J Dent Res. 2015;94(7):9907.

45. Cao X, Han Y, Gu M, Du H, Song M, Zhu X, et al. Foodborne Titanium Dioxide Nanoparticles Induce Stronger Adverse Effects in Obese Mice than Non-Obese Mice: Gut Microbiota Dysbiosis, Colonic Inflammation, and Proteome Alterations. Small. 2020;16(36):2001858.

46. De Munck TJI, Xu P, Verwijs HJA, Masclee AAM, Jonkers D, Verbeek J, et al. Intestinal permeability in human nonalcoholic fatty liver disease: A systematic review and meta-analysis. Liver Int. 2020;40(12):2906-16. 
47. Chopyk DM, Grakoui A. Contribution of the Intestinal Microbiome and Gut Barrier to Hepatic Disorders. Gastroenterology. 2020;159(3):849-863.

48. Paone P, Cani PD. Mucus barrier, mucins and gut microbiota: the expected slimy partners? Gut. 2020;69(12):2232-2243.

49. Wang W, Zhai S, Xia Y, Wang H, Ruan D, Zhou T, et al. Ochratoxin A induces liver inflammation: involvement of intestinal microbiota. Microbiome. 2019;7(1):151.

50. Cho Y, Kim D, Seo W, Gao B, Yoo S, Song B. Fructose Promotes Leaky Gut, Endotoxemia, and Liver Fibrosis Through Ethanol-Inducible Cytochrome P450-2E1-Mediated Oxidative and Nitrative Stress. Hepatology. 2021;73(6):2180-2195.

51. Amadeu De Oliveira F, Macvinish LJ, Amin S, Herath D, Jeggle P, Mela I, et al. The effect of fluoride on the structure, function, and proteome of intestinal epithelia. Environ Toxicol. 2018;33(1):63-71.

52. Miao L, Gong Y, Li H, Xie C, Xu Q, Dong X, et al. Alterations in cecal microbiota and intestinal barrier function of laying hens fed on fluoride supplemented diets. Ecotox Environ Safe. 2020;193:110372.

53. Yan X, Chen X, Tian X, Qiu Y, Wang J, Yu G, et al. Co-exposure to inorganic arsenic and fluoride prominently disrupts gut microbiota equilibrium and induces adverse cardiovascular effects in offspring rats. Sci Total Environ. 2021;767:144924.

54. Ziętak M, Kovatcheva-Datchary P, Markiewicz LH, StåhIman M, Kozak LP, Bäckhed F. Altered Microbiota Contributes to Reduced Diet-Induced Obesity upon Cold Exposure. Cell Metab. 2016;23(6):1216-23.

55. Martínez I, Perdicaro DJ, Brown AW, Hammons S, Carden TJ, Carr TP, et al. Diet-Induced Alterations of Host Cholesterol Metabolism Are Likely To Affect the Gut Microbiota Composition in Hamsters. Appl Environ Microb. 2013;79(2):516-24.

56. Kaakoush NO. Insights into the Role of Erysipelotrichaceae in the Human Host. Front Cell Infect Mi. 2015;5:84.

57. Yasuda K, Hsu T, Gallini CA, Mclver LJ, Schwager E, Shi A, et al. Fluoride Depletes Acidogenic Taxa in Oral but Not Gut Microbial Communities in Mice. mSystems. 2017;2(4):e17-47.

58. Roager HM, Vogt JK, Kristensen M, Hansen LBS, Ibrügger S, Mærkedahl RB, et al. Whole grain-rich diet reduces body weight and systemic low-grade inflammation without inducing major changes of the gut microbiome: a randomised cross-over trial. Gut. 2019;68(1):83-93.

59. Aron-Wisnewsky J, Prifti E, Belda E, Ichou F, Kayser BD, Dao MC, et al. Major microbiota dysbiosis in severe obesity: fate after bariatric surgery. Gut. 2019;68(1):70-82.

60. Woting A, Pfeiffer N, Loh G, Klaus S, Blaut M. Clostridium ramosum Promotes High-Fat Diet-Induced Obesity in Gnotobiotic Mouse Models. mBio. 2014;5(5):e1514-30.

61. Fujimoto K, Kawaguchi Y, Shimohigoshi M, Gotoh Y, Nakano Y, Usui Y, et al. Antigen-Specific Mucosal Immunity Regulates Development of Intestinal Bacteria-Mediated Diseases. Gastroenterology. 2019;157(6):1530-1543. 
62. Zuo T, Zhang F, Lui GCY, Yeoh YK, Li AYL, Zhan H, et al. Alterations in Gut Microbiota of Patients With COVID-19 During Time of Hospitalization. Gastroenterology. 2020;159(3):944-55.

63. Wu Y, Jiao N, Zhu R, Zhang Y, Wu D, Wang A, et al. Identification of microbial markers across populations in early detection of colorectal cancer. Nat Commun. 2021;12(1):3063.

64. Verspreet J, Damen B, Broekaert WF, Verbeke K, Delcour JA, Courtin CM. A Critical Look at Prebiotics Within the Dietary Fiber Concept. Annu Rev Food Sci T. 2016;7(1):167-90.

65. Makki K, Deehan EC, Walter J, Bäckhed F. The Impact of Dietary Fiber on Gut Microbiota in Host Health and Disease. Cell Host Microbe. 2018;23(6):705-15.

66. Umarani V, Muvvala S, Ramesh A, Lakshmi BVS, Sravanthi N. Rutin potentially attenuates fluorideinduced oxidative stress-mediated cardiotoxicity, blood toxicity and dyslipidemia in rats. Toxicol Mech Method. 2015;25(2):143-9.

67. Yang $\mathrm{H}$, Wang W, Romano KA, Gu M, Sanidad KZ, Kim D, et al. A common antimicrobial additive increases colonic inflammation and colitis-associated colon tumorigenesis in mice. Sci Transl Med. 2018;10(443):n4116.

68. Rizvi ZA, Dalal R, Sadhu S, Kumar Y, Kumar S, Gupta SK, et al. High-salt diet mediates interplay between NK cells and gut microbiota to induce potent tumor immunity. Sci Adv. 2021;7(37):g5016.

69. Jensen BAH, Holm JB, Larsen IS, von Burg N, Derer S, Sonne SB, et al. Lysates of Methylococcus capsulatus Bath induce a lean-like microbiota, intestinal FoxP3 ${ }^{+} \mathrm{RORyt} \mathrm{I}^{+} \mathrm{IL}-17^{+}$Tregs and improve metabolism. Nat Commun. 2021;12(1):1093.

70. Liu Y, Yang K, Jia Y, Shi J, Tong Z, Fang D, et al. Gut microbiome alterations in high-fat-diet-fed mice are associated with antibiotic tolerance. Nat Microbiol. 2021;6(7):874-84.

71. Peng Y, Xi J, Sun Y, Chen G, Li D, Peng C, et al. Tea components influencing bioavailability of fluoride and potential transport mechanism in the Caco-2 cell line model. Int J Food Sci Technol. 2020;55(4):1792-9.

72. Wu T, Lin C, Chang C, Lin T, Martel J, Ko Y, et al. Gut commensal Parabacteroides goldsteinii plays a predominant role in the anti-obesity effects of polysaccharides isolated from Hirsutella sinensis. Gut. 2019;68(2):248-262.

73. Gu L, Cai N, Lyu Y, Yao L, Wang F, Xu H, et al. Y-Mangostin Ameliorates Free Fatty Acid-Induced Lipid Accumulation via the SIRT1/LKB1/AMPK Pathway in HepG2 and L02 Cells. J Agr Food Chem. 2019;67(50):13929-38.

74. Peng Y, Yan Y, Wan P, Chen D, Ding Y, Ran L, et al. Gut microbiota modulation and anti-inflammatory properties of anthocyanins from the fruits of Lycium ruthenicum Murray in dextran sodium sulfateinduced colitis in mice. Free Radical Bio Med. 2019;136:96-108.

75. Jensen SN, Cady NM, Shahi SK, Peterson SR, Gupta A, Gibson-Corley KN, et al. Isoflavone diet ameliorates experimental autoimmune encephalomyelitis through modulation of gut bacteria depleted in patients with multiple sclerosis. Sci Adv. 2021;7(28):d4595. 
76. Zhou Q, Zhang Y, Wang X, Yang R, Zhu X, Zhang Y, et al. Gut bacteria Akkermansia is associated with reduced risk of obesity: evidence from the American Gut Project. Nutr Metab. 2020;17(1):90.

77. Rodrigues RR, Gurung M, Li Z, García-Jaramillo M, Greer R, Gaulke C, et al. Transkingdom interactions between Lactobacilli and hepatic mitochondria attenuate western diet-induced diabetes. Nat Commun. 2021;12(1):101.

78. Liu Y, Qin Y, Chen T, Lu M, Qian X, Guo X, et al. A practical guide to amplicon and metagenomic analysis of microbiome data. Protein Cell. 2021;12(5):315-30.

\section{Figures}
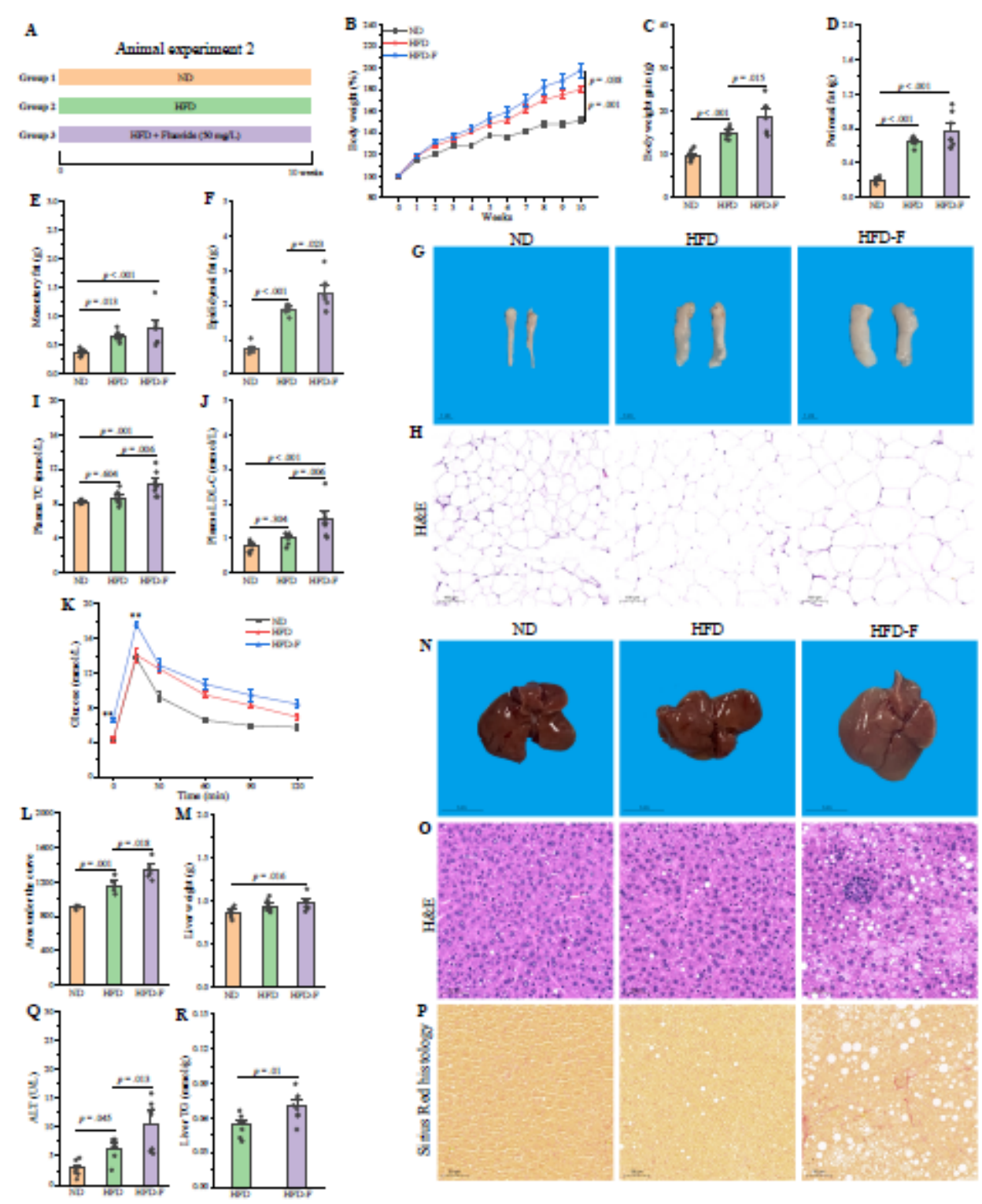

Figure 1

Fluoride exacerbates the obesity in HFD mice. (A) Scheme of animal experiment 2 over the 10 weeks of dietary intervention. Mice were randomly divided into three groups including ND group, HFD group, and 
HFD plus $50 \mathrm{mg} / \mathrm{L}$ of fluoride in drinking water (HFD-F) group $(\mathrm{n}=8)$. (B) Dynamic changes in body weight in mice. (C) Body weight gain. (D) Perirenal fat. (E) Mesentery fat. (F) Epididymal fat. (G-H) Representative morphology and H\&E staining of Epididymal fat. (I-J) Plasma levels of TC and LDL-C. (K) OGTT carried out at week 9, mice were fasted overnight and gavaged with a dosage of glucose with 1.5 $\mathrm{mg} / \mathrm{g}$ body weight ( $\mathrm{n}=5$ per group). (L) AUC for OGTT. (M) Liver weight. (N-P) Representative morphology, H\&E staining and Sirius Red histology of liver. (Q) Plasma ALT. (R) Liver TG. The results were expressed as means \pm SEM. Statistical significance was carried out by one-way analysis of variance (ANOVA) with Tukey test for multiple-group comparisons. Difference in two groups was calculated using the Mann-Whitney test or Kruskal-Wallis test. A value of $p<0.05$ was considered to be significant.

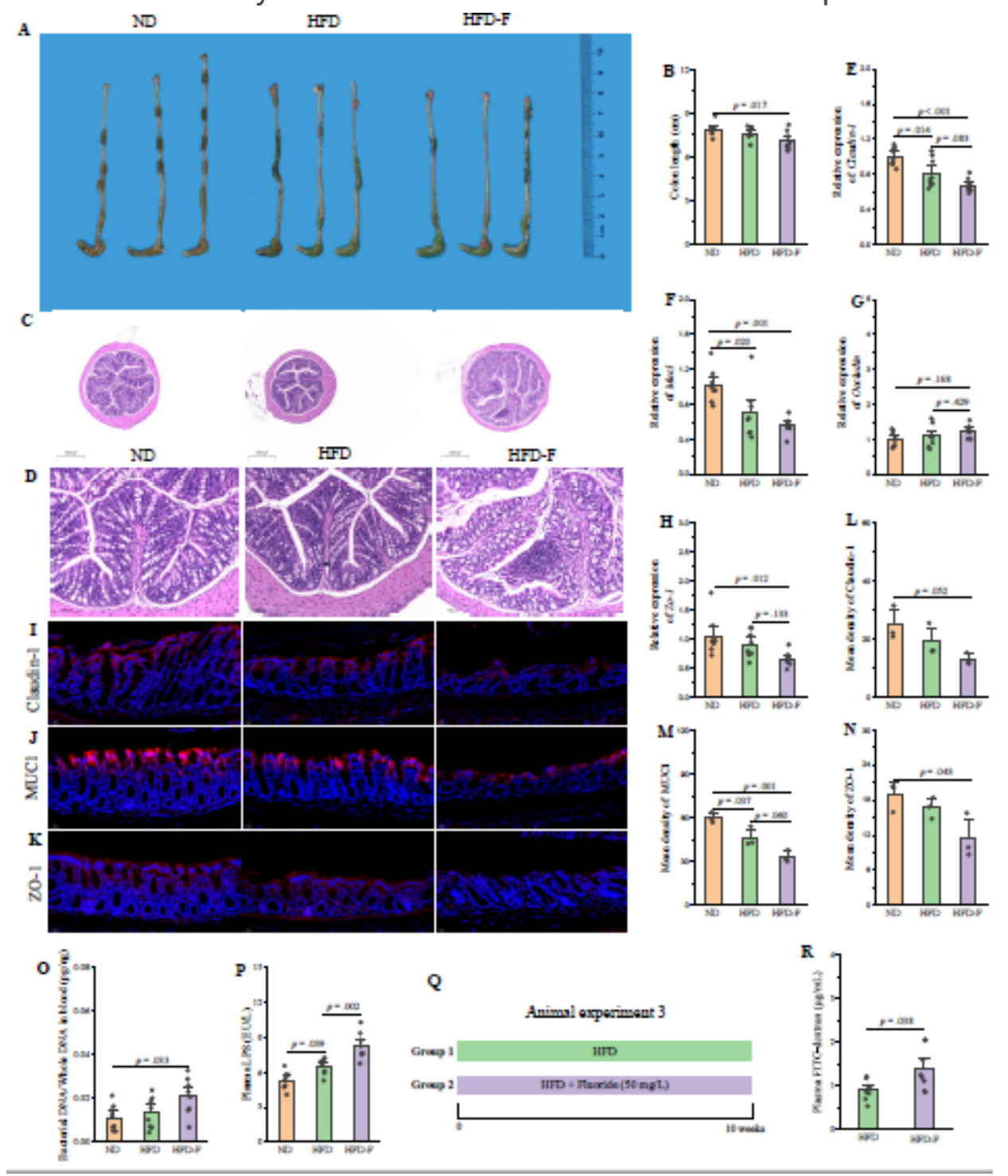

\section{Figure 2}

Fluoride drives the intestinal barrier permeability in HFD mice. (A) Representative morphology of colon. (B) Colon length $(n=8)$. (C-D) Representative H\&E staining of colon (10 $\times$ and $40 \times$, respectively). $(E-H)$ Relative mRNA expression levels of Claudin-1, Muc1, Occludin and Zo-1 in colon $(n=8)$. (I-K) Representative immunofluorescence images of Claudin-1, MUC1 and ZO-1 in colon. (L-N) Mean density of Claudin-1, MUC1 and ZO-1 in immunofluorescence images was evaluated by ImageJ software $(n=3)$. 
Bacterial DNA/Whole DNA in blood was measured by RT-qPCR $(n=8)$. (P) Plasma LPS. (Q) Scheme of animal experiment 3. (R) Plasma FITC-dextran from mice in animal experiment 3 was measured after $4 \mathrm{~h}$ of intragastric administration of FITC-dextran $(n=8)$. The results were expressed as means \pm SEM. Statistical significance was carried out by one-way ANOVA with Tukey test for multiple-group comparisons. Difference in two groups was calculated using the Mann- Whitney test or Kruskal-Wallis test. A value of $p<0.05$ was considered to be significant.

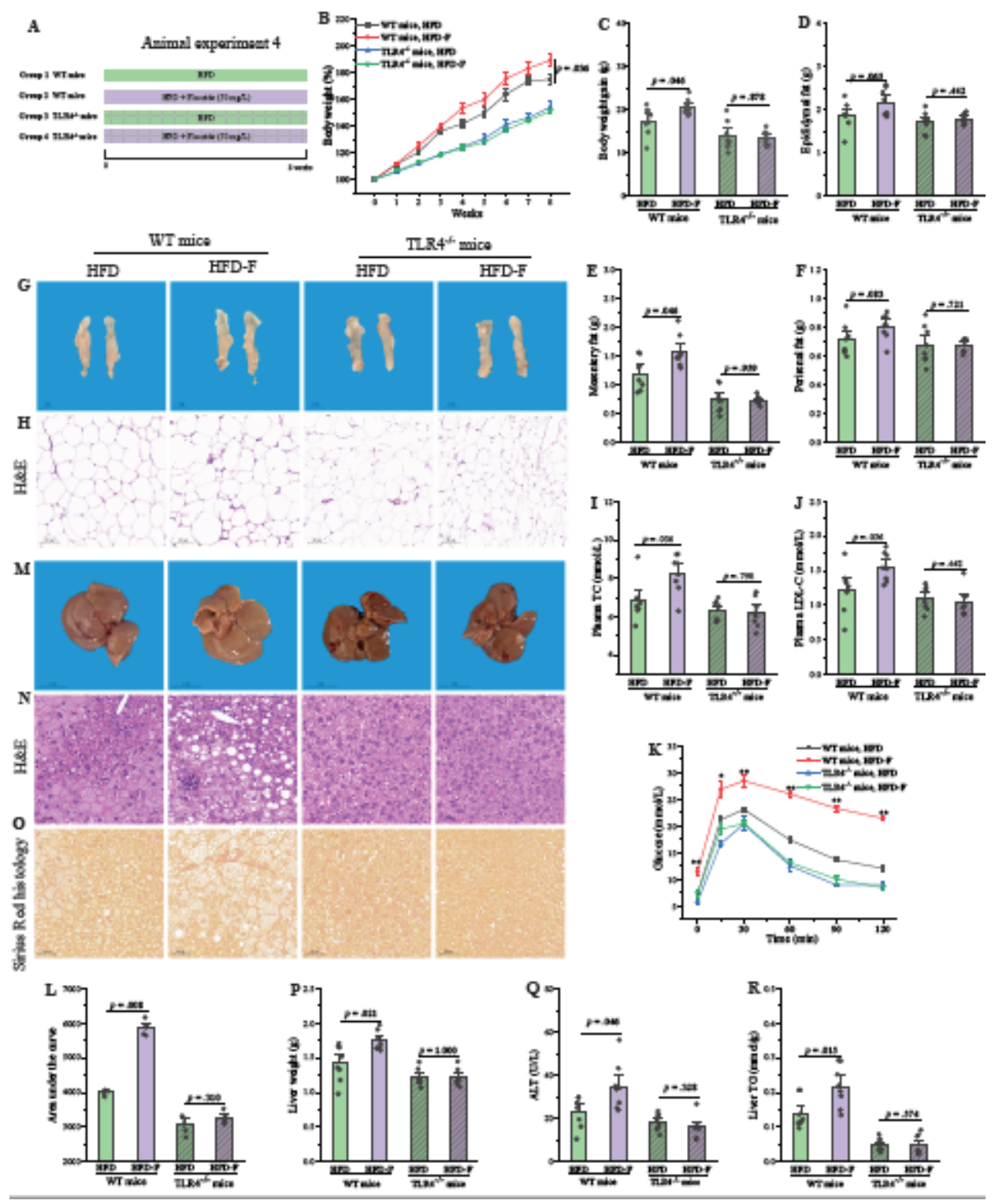

Figure 3

Fluoride exacerbates the obesity in HFD mice through a TLR4-dependent mechanism. (A) Scheme of animal experiment 4 over the 8 weeks of dietary intervention. Wild-type (WT) mice and TIr4 knockout (TIr4-/-) mice were randomly divided into two groups, respectively, including HFD group, and HFD-F groups ( $\mathrm{n}=8$ per group). (B) Dynamic changes in body weight in mice. (C) Body weight gain. (D) Epididymal fat. (E)Mesentery fat. (F) Perirenal fat. (G-H) Representative morphology and H\&E staining of Epididymal fat. (I-J) Plasma levels of TC and LDL-C. (K) OGTT was carried out at week 7, mice were 
fasted overnight and gavaged with a dosage of glucose with $1.5 \mathrm{mg} / \mathrm{g}$ body weight ( $\mathrm{n}=5$ per group). (L) AUC for OGTT. (M-O) Representative morphology, H\&E staining and Sirius Red histology of liver. (P) Liver weight. (Q) Plasma ALT. (R) Liver TG. (S-U) The plasma levels of TNF-a, IL-6, and IL-1 $\beta$. The results were expressed as means \pm SEM. Difference in two groups was calculated using the Mann-Whitney test or Kruskal-Wallis test. A value of $p<0.05$ was considered to be significant.
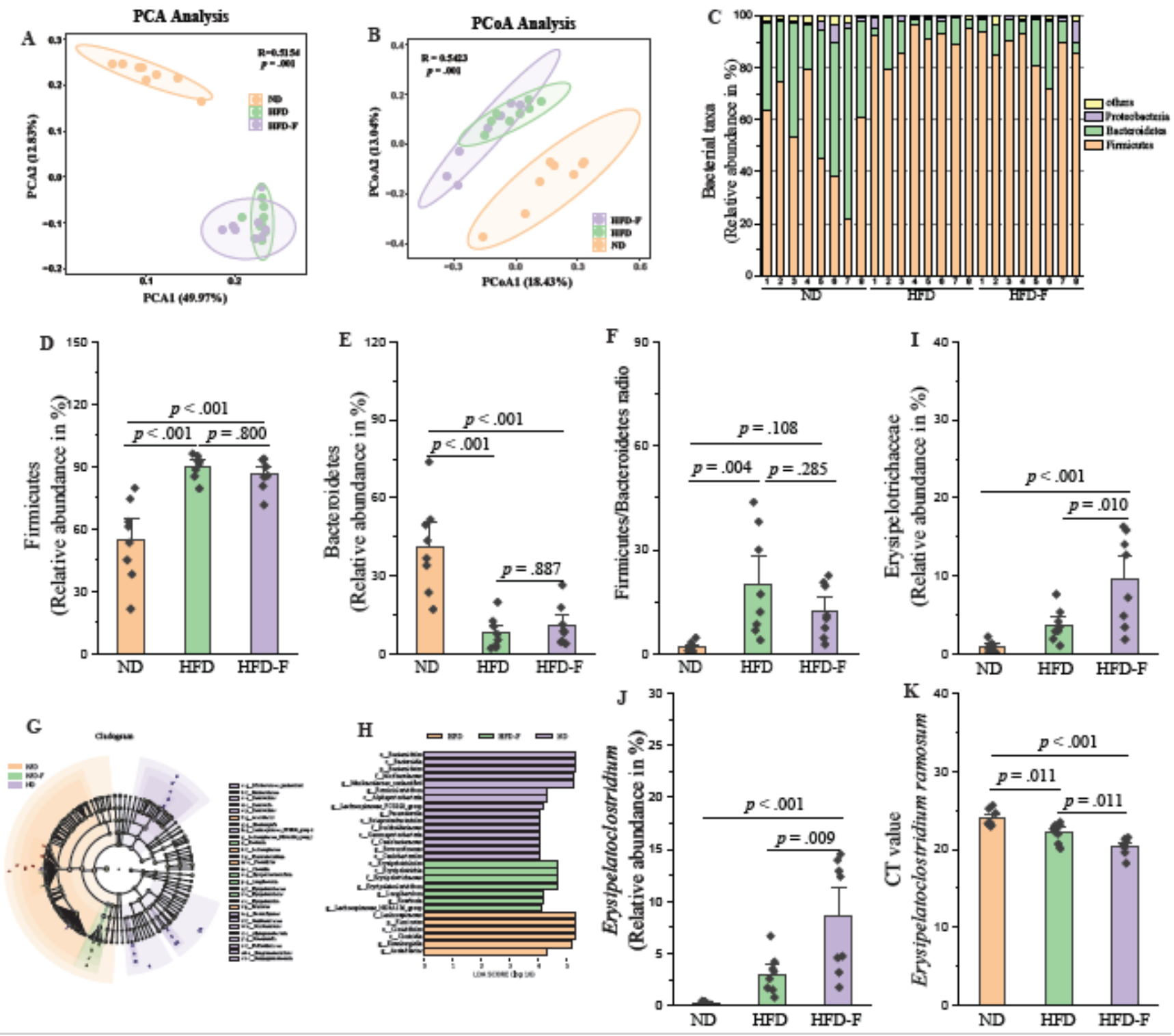

Figure 4

Fluoride disturbs the gut microbiota in HFD mice. Gut microbiota was analyzed by 16S rRNA gene sequencing ( $n=8$ for each group). (A) PCA based on the relative abundance of Features of the gut microbiota, (B) PCoA of the gut microbiota based on the unweighted unifrac distance matrix, (C) Bacterial taxonomic profiling at the phylum level of gut microbiota, the relative abundances of $(D)$ Firmicutes, $(E)$ Bacteroidetes, and (F) the ratio of Firmicutes to Bacteroidetes. (G-H) LEfSe analyses of gut microbiota (value of Kruskal-Wallis: 0.01, value of Wilcoxon: 0.01, value of LDA score: 4). the relative abundances of (I) Erysipelotrichaceae, and (J) Erysipelatoclostridium, (K) Level of E. ramosum in fecal samples measured by RTqPCR using species-specific primers. Higher CT values suggest lower levels of E. 
ramosum. The results were expressed as means \pm SEM. Statistical significance was carried out by oneway ANOVA with Tukey test. A value of $p<0.05$ was considered to be significant.
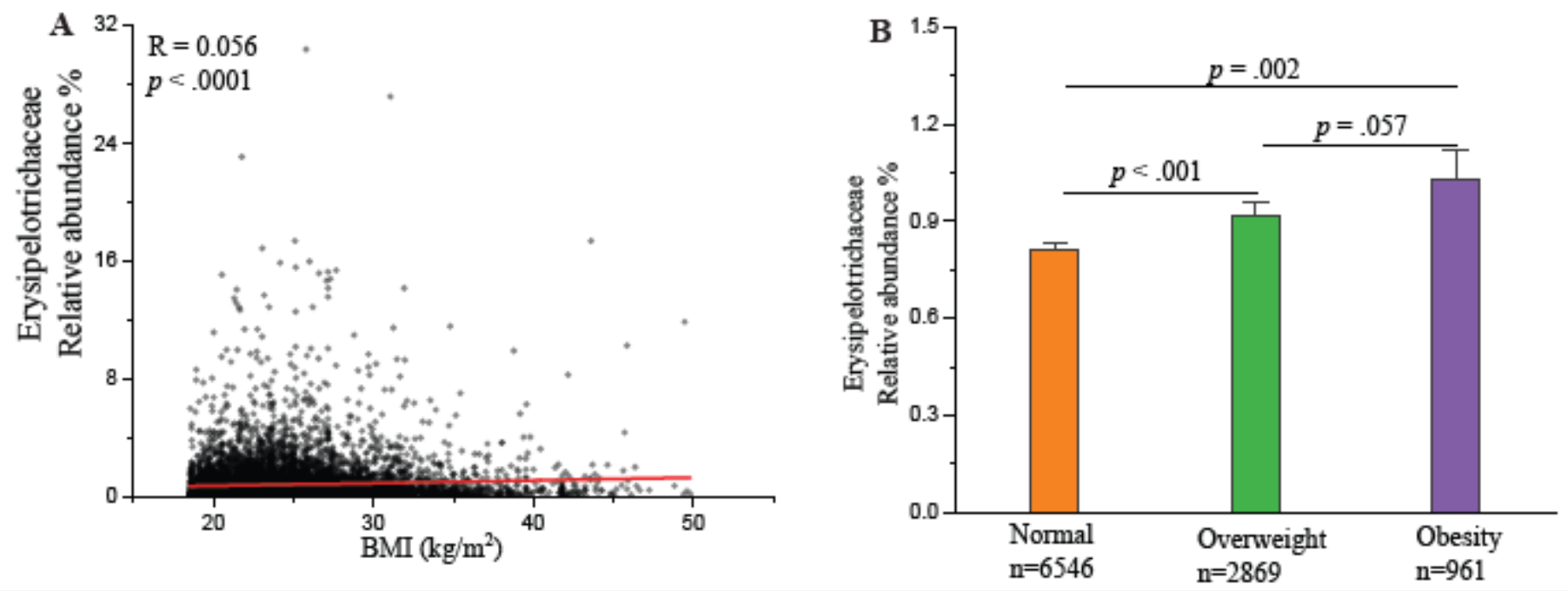

Figure 5

The association between Erysipelotrichaceae and BMI based on the database of American Gut Project (AGP). (A) Pearson correlation between the relative abundance of Erysipelotrichaceae and BMI ( $n=$ 10376). (B) The relative abundance of Erysipelotrichaceae in three BMI categories, namely Normal (18.5 $\leq \mathrm{BMI}<25, \mathrm{n}=6546)$, Overweight $(25 \leq \mathrm{BMI}<30, \mathrm{n}=2869)$, and Obesity $(\mathrm{BMI} \geq 30, \mathrm{n}=961)$. The results were expressed as means \pm SEM. Statistical significance was carried out by one-way ANOVA with Tukey test. A value of $p<.05$ was considered to be significant. 


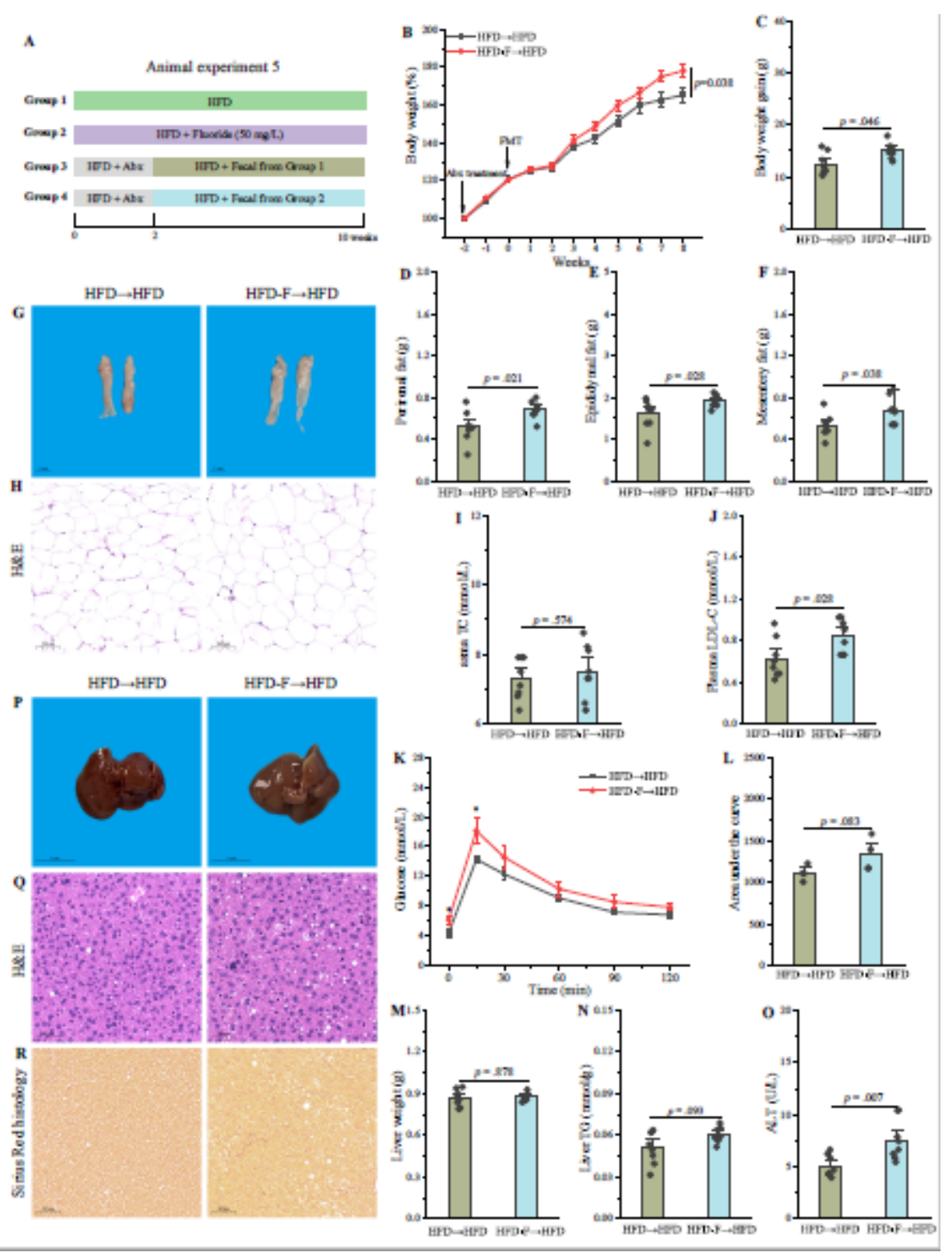

Figure 6

FMT is sufficient to induce some phenotypical changes caused by fluoride in a HFD context. (A) Scheme of animal experiment 5 over the 10 weeks. Pseudogermfree mice, induced by Abx for two weeks, were colonized by fecal slurry from either HFD group or HFD-F group mice $(n=8)$ for 8 weeks. (B) Dynamic changes in body weight in mice. (C) Body weight gain. (D) Perirenal fat. (E) Epididymal fat. (F) Mesentery fat. (G-H) Representative morphology and H\&E staining of Epididymal fat. (I-J) Plasma levels of TC and LDL-C. (K) OGTT was carried out at week 9, mice were fasted overnight and gavaged with a dosage of glucose with $1.5 \mathrm{mg} / \mathrm{g}$ body weight ( $\mathrm{n}=4$ per group). (L) AUC for OGTT. (M) Liver weight. (N) Liver TG. (O) Plasma ALT. (P-R) Representative morphology, H\&E staining and Sirius Red histology of liver. The results were expressed as means \pm SEM. Difference in two groups was calculated using the MannWhitney test or Kruskal-Wallis test. A value of $p<0.05$ was considered to be significant. 

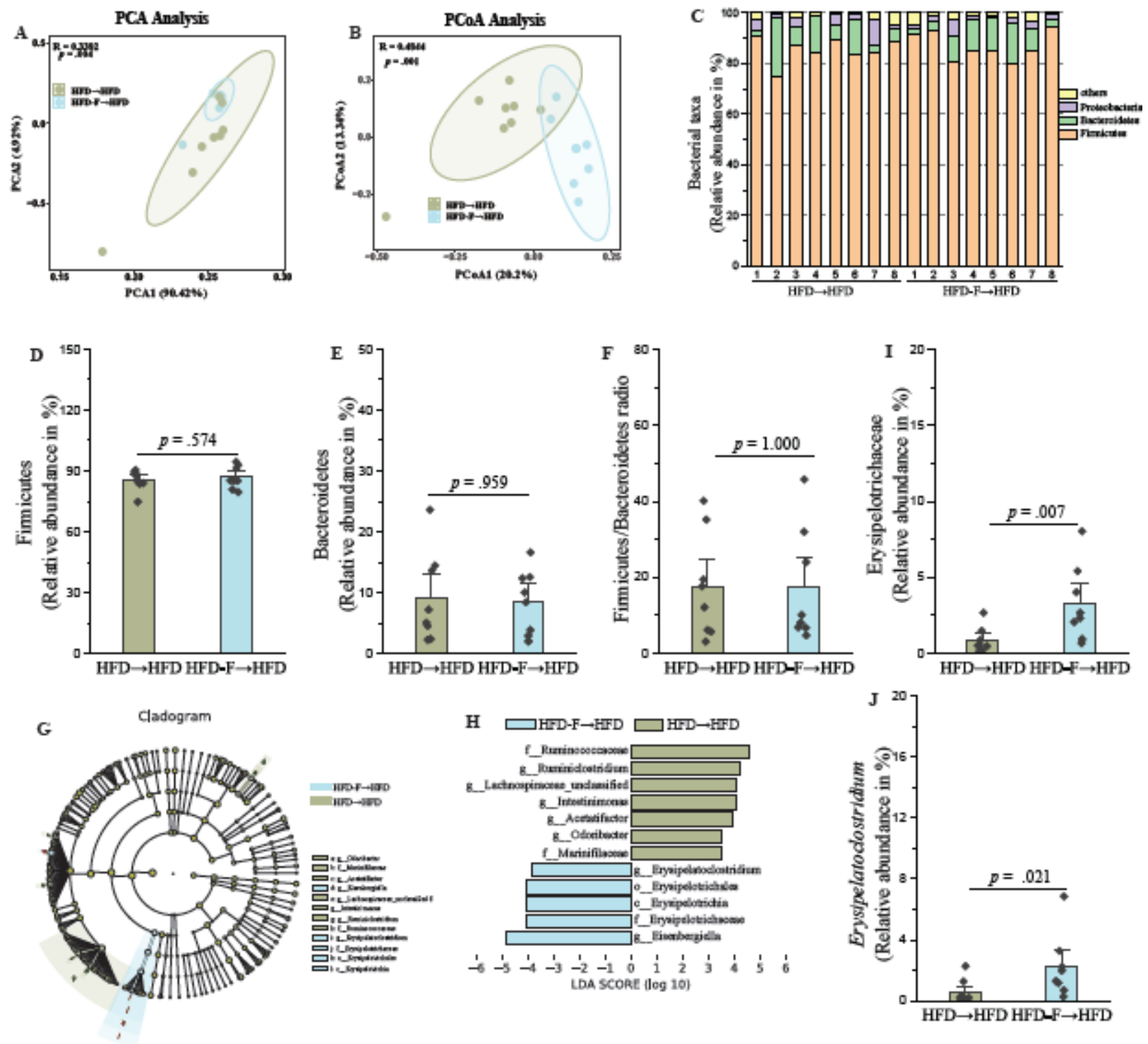

\section{Figure 7}

The fluoride-induced key gut microbiota could colonize in pseudo-germfree mice by FMT. Gut microbiota was analyzed by $16 \mathrm{~S}$ rRNA gene sequencing ( $\mathrm{n}=8$ for each group). (A) PCA based on the relative abundance of Features of the gut microbiota, (B) PCoA of the gut microbiota based on the unweighted unifrac distance matrix, (C) Bacterial taxonomic profiling at the phylum level of gut microbiota, the relative abundances of (D) Firmicutes, (E) Bacteroidetes, and (F) the ratio of Firmicutes to Bacteroidetes. (G-H) LEfSe analyses of gut microbiota (value of Kruskal-Wallis: 0.05 , value of Wilcoxon: 0.05 , value of LDA score: 3.5 ). the relative abundances of (I) Erysipelotrichaceae, and (J) Erysipelatoclostridium. The results were expressed as means \pm SEM. Difference in two groups was calculated using the MannWhitney test or Kruskal-Wallis test. A value of $p<0.05$ was considered to be significant. 
A
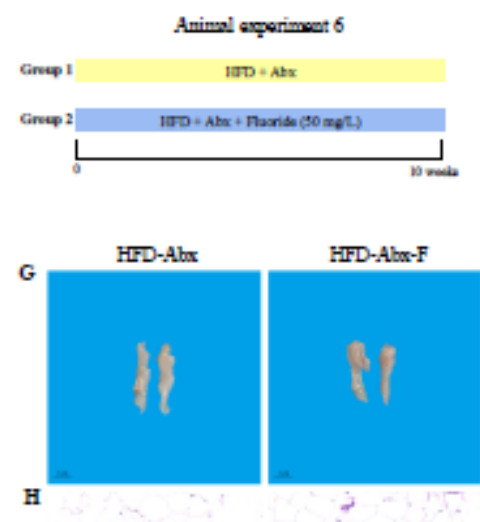

옆

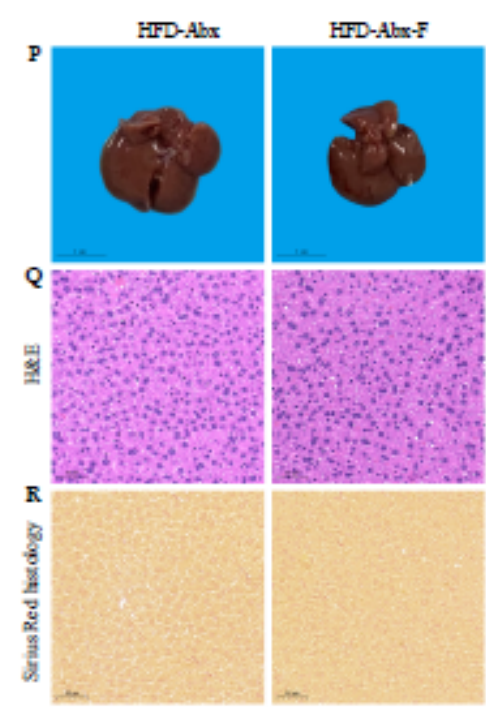

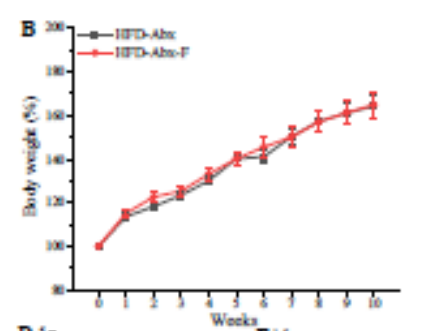
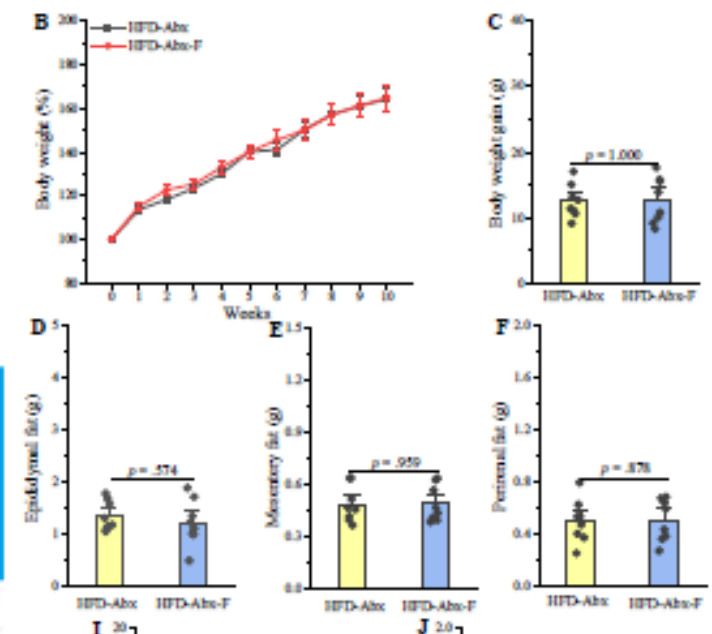

$3^{20}$

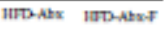
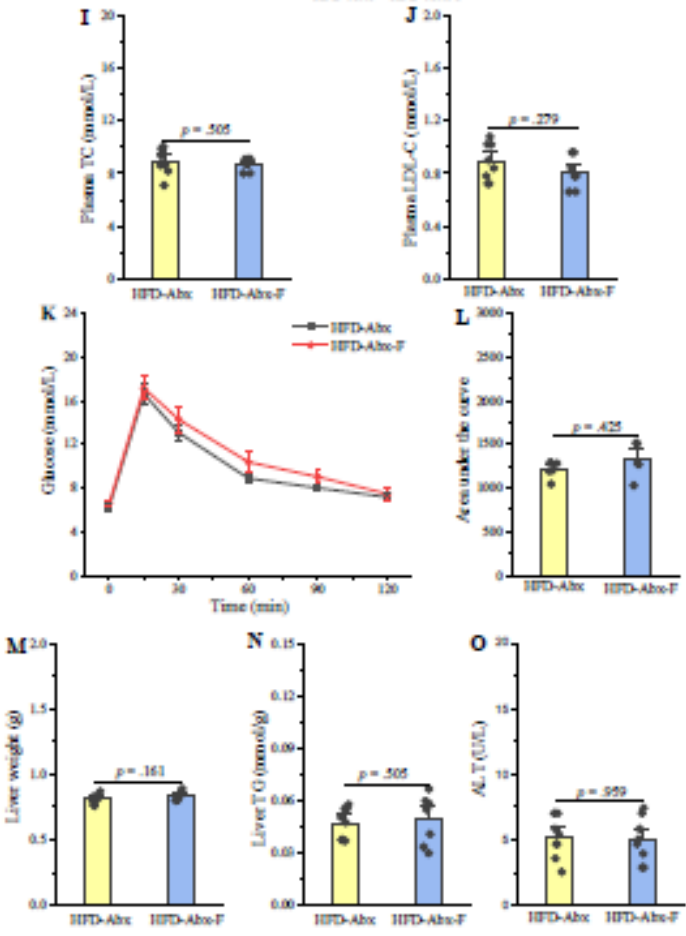

\section{Figure 8}

The exacerbation of the obesity by fluoride is blocked after depletion of the gut microbiota by Abx. (A) Scheme of animal experiment 6 over the 10 weeks. Mice were randomly divided into two groups including HFD fed with Abx (HFD-Abx) group, and HFD fed with Abx plus $50 \mathrm{mg} / \mathrm{L}$ of fluoride in drinking water (HFD-Abx-F) group ( $\mathrm{n}=8$ per group). Abx was fed by intragastric gavage. (B) Dynamic changes in body weight in mice. (C) Body weight gain. (D) Epididymal fat. (E) Mesentery fat. (F) Perirenal fat. (G-H) Representative morphology and H\&E staining of Epididymal fat. (I-J) Plasma levels of TC and LDL-C. (K) OGTT was carried out at week 9, mice were fasted overnight and gavaged with a dosage of glucose with $1.5 \mathrm{mg} / \mathrm{g}$ body weight ( $\mathrm{n}=5$ per group). (L) AUC for OGTT. (M) Liver weight. (N) Liver TG. (O) Plasma ALT. (P-R) Representative morphology, H\&E staining and Sirius Red histology of liver. The results were expressed as means \pm SEM. Difference in two groups was calculated using the Mann-Whitney test or Kruskal-Wallis test. A value of $p<0.05$ was considered to be significant. 
A
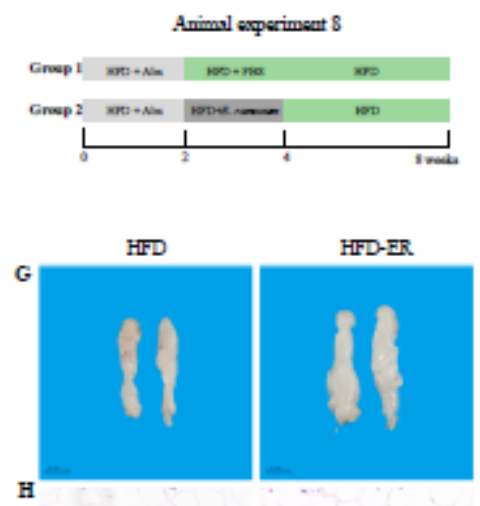

HFD-ER

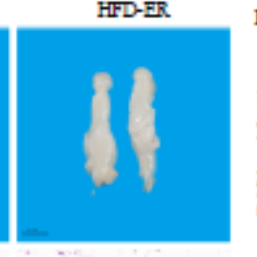

뽄

HrD

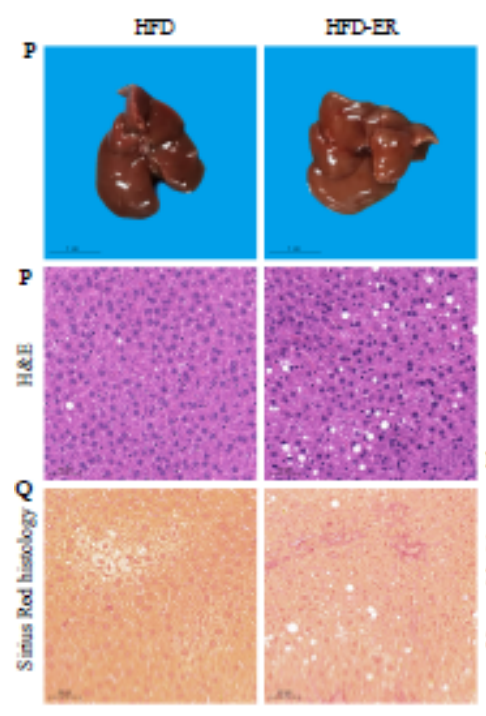

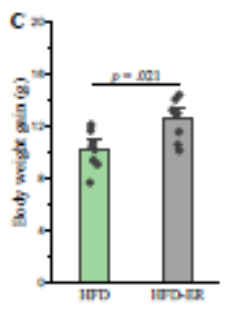
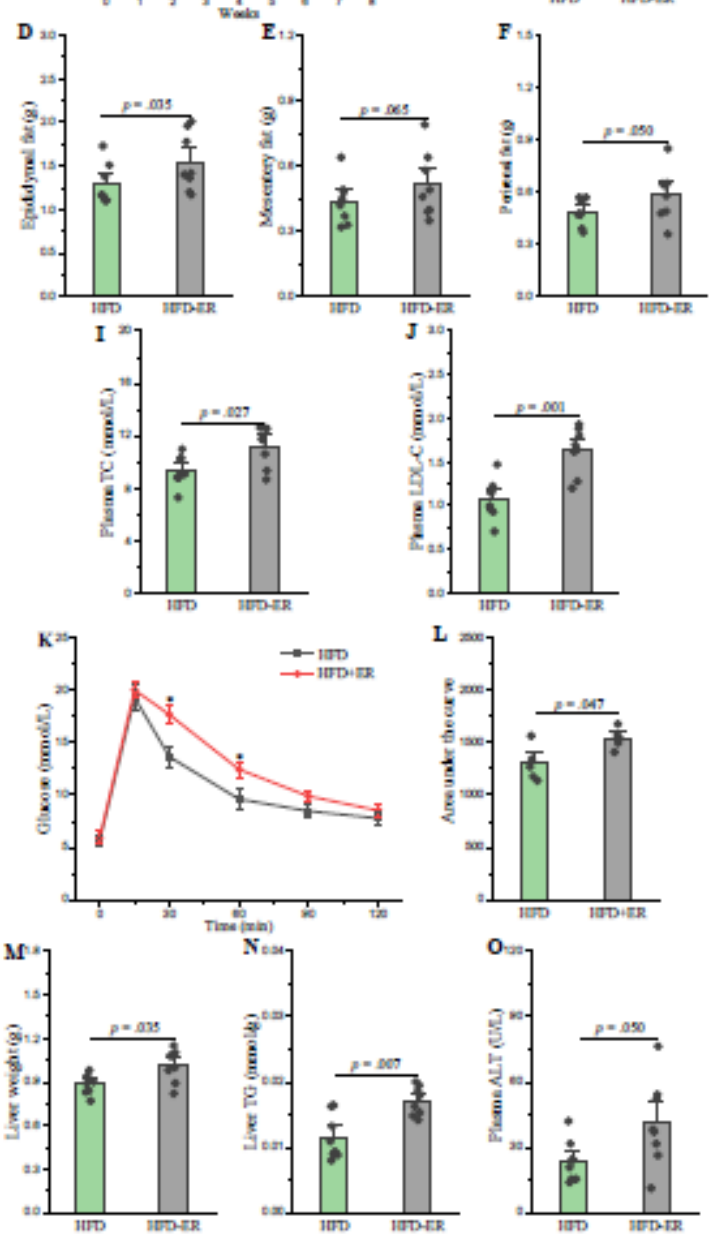

Figure 9

E. ramosum aggravates the obesity in HFD mice. (A) Scheme of animal experiment 8 over the 8 weeks. Pseudo-germfree mice were obtained by treatment of Abx for two weeks. Then, pseudo-germfree mice were randomly divided into two groups including HFD fed with PBS and HFD fed with E. ramosum (HFDER) by intragastric gavage for two weeks. (B) Dynamic changes in body weight in mice. (C) Body weight gain. (D) Epididymal fat. (E) Mesentery fat. (F) Perirenal fat. (G-H) Representative morphology and H\&E staining of Epididymal fat. (I-J) Plasma levels of TC and LDL-C. (K) OGTT was carried out at week 7, mice were fasted overnight and gavaged with a dosage of glucose with $1.5 \mathrm{mg} / \mathrm{g}$ body weight $(\mathrm{n}=5$ per group). (L) AUC for OGTT. (M) Liver weight. (N) Liver TG. (O) Plasma ALT. (P-R) Representative morphology, H\&E staining and Sirius Red histology of liver. The results were expressed as means \pm SEM. Difference in two groups was calculated using the Mann-Whitney test or Kruskal-Wallis test. A value of $p$ $<0.05$ was considered to be significant. 


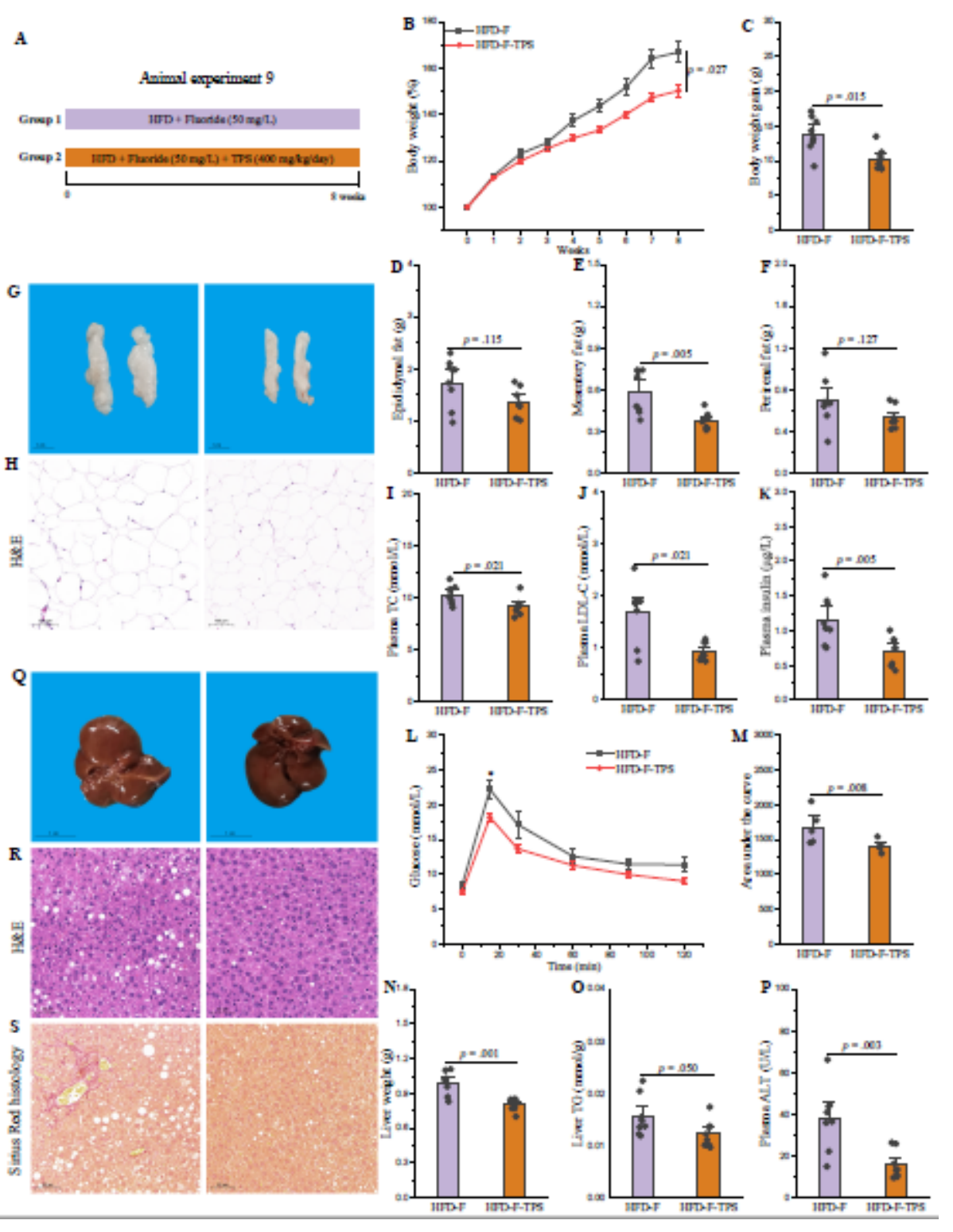

Figure 10

FBTPS abolish the fluoride-induced obesity in HFD mice. (A) Scheme of animal experiment 9 over the 8 weeks. Mice were randomly divided into two groups including HFD plus $50 \mathrm{mg} / \mathrm{L}$ of fluoride in drinking water (HFD-F) group, and HFD plus $50 \mathrm{mg} / \mathrm{L}$ of fluoride in drinking water and $400 \mathrm{mg} / \mathrm{kg} / \mathrm{day}$ of TPS from Fuzhuan brick by intragastric gavage (HFD-F-TPS) group ( $\mathrm{n}=8$ per group). (B) Dynamic changes in body weight in mice. (C) Body weight gain. (D) Epididymal fat. (E) Mesentery fat. (F) Perirenal fat. (G-H) Representative morphology and H\&E staining of Epididymal fat. (I-K) Plasma levels of TC, LDL-C and insulin. (L) OGTT was carried out at week 7, mice were fasted overnight and gavaged with a dosage of glucose with $1.5 \mathrm{mg} / \mathrm{g}$ body weight ( $\mathrm{n}=5$ per group). (M) AUC for OGTT. (N) Liver weight. (0) Liver TG. (P) Plasma ALT. (Q-S) Representative morphology, H\&E staining and Sirius Red histology of liver. The results were expressed as means \pm SEM. Difference in two groups was calculated using the MannWhitney test or Kruskal-Wallis test. A value of $p<0.05$ was considered to be significant. 


\section{Supplementary Files}

This is a list of supplementary files associated with this preprint. Click to download.

- Additionalfile1.pdf 